\title{
Relocating a Cluster of Earthquakes Using a Single Seismic Station
}

\author{
by David J. Robinson," Malcolm Sambridge, Roel Snieder, and Juerg Hauser
}

\begin{abstract}
Coda waves arise from scattering to form the later arriving components of a seismogram. Coda-wave interferometry (CWI) is an emerging tool for constraining earthquake source properties from the interference pattern of coda waves between nearby events. A new earthquake location algorithm is derived which relies on coda-wave-based probabilistic estimates of earthquake separation. The algorithm can be used with coda waves alone or in tandem with arrival-time data. Synthetic examples (2D and 3D) and real earthquakes on the Calaveras fault, California, are used to demonstrate the potential of coda waves for locating poorly recorded earthquakes. It is demonstrated that CWI: (a) outperforms traditional earthquake location techniques when the number of stations is small; (b) is self-consistent across a broad range of station situations; and (c) can be used with a single station to locate earthquakes.
\end{abstract}

\section{Introduction}

Accurate earthquake location is important for many applications. Locations are required for: magnitude determination (Richter, 1935; Gutenberg, 1945); computing moment tensors (Sipkin, 2002); seismological studies of the Earth's interior (Spencer and Gubbins, 1980; Kennett et al., 1995, 2004; Curtis and Snieder, 2002); understanding strong motion and seismic attenuation (Toro et al., 1997; Campbell, 2003); and modeling earthquake hazard or risk (Frankel et al., 2000; Stirling et al., 2002; Robinson et al., 2006). The accuracy required in earthquake location depends on the application. For example, imaging the structure of a fracture system from microseismicity requires greater location accuracy than determining whether an $M_{\mathrm{w}} 7.5$ earthquake occurs offshore for tsunami warning. This paper focuses on reducing location uncertainty for a cluster of events when they are recorded by a small number of stations.

Absolute location describes the location of an earthquake with respect to a global reference such as latitude, longitude (or easting/northing), and depth. Uncertainties associated with absolute locations are influenced by source to station distances, the number of stations and their geometry, signal-to-noise ratio, clarity of onsets, and accuracy of the velocity model used in computing arrival times. Uncertainties in absolute location are typically of the order of several kilometers, primarily because they are susceptible to uncertainty in the velocity structure along the entire path between the source and receiver. For example, Shearer (1999) states that location uncertainties in the International Sesimological Centre (ISC) and the National Earthquake Information Center's Preliminary Determination of Epicenters (PDE)

\footnotetext{
*Also at Research School of Earth Sciences, Australian National University, Canberra ACT 0200, Australia.
}

databases are generally around $25 \mathrm{~km}$ horizontally and at least $25 \mathrm{~km}$ in depth. (Here the depth uncertainties of $25 \mathrm{~km}$ assume the use of depth-dependent phases such as $p P$. Without such phases the uncertainty is higher.) Bondár et al. (2004) demonstrate that absolute locations are accurate to within $5 \mathrm{~km}$ with a 95\% confidence level when local networks meet the following criteria:

1. there are 10 or more stations, all within $250 \mathrm{~km}$,

2. an azimuthal gap of less than $110^{\circ}$,

3. a secondary azimuthal gap of less than $160^{\circ}$, and

4. at least one station within $30 \mathrm{~km}$.

Such errors are too large for many applications, particularly those focused on imaging rupture surfaces from aftershock sequences.

Relative earthquake location involves locating a group of earthquakes with respect to one another and was first introduced by Douglas (1967) who developed the technique commonly known as joint hypocenter determination. (Douglas, 1967, originally used the term "joint epicentre determination"; however, he was solving for hypocenter.) In principle, relative locations can be computed by differencing absolute locations. However, Pavlis (1992) shows that inadequate knowledge of velocity structure leads to systematic biases when relative positions are computed in this way. To reduce errors from unknown velocity structure, relative location techniques typically compute locations directly from arrival-time differences computed by time-lag cross correlation of early-onset body waves (Ito, 1985; Got et al., 1994; Slunga et al., 1995; Nadeau and McEvilly, 1997; Waldhauser et al., 1999). Doing so removes errors associated with velocity variations outside the local region, because such variations influence all waveforms in a similar manner (Shearer, 1999). 
Reported location uncertainties from relative techniques are around 15-75 $\mathrm{m}$ in local settings with good station coverage (Ito, 1985; Got et al., 1994; Waldhauser et al., 1999; Waldhauser and Schaff, 2008). Here, "good coverage" implies multiple stations distributed across a broad range of azimuthal directions. Relative location techniques have been used to image active fault planes (Deichmann and GarciaFernandez, 1992; Got et al., 1994; Waldhauser et al., 1999; Waldhauser and Ellsworth, 2002; Shearer et al., 2005); study rupture mechanics (Rubin et al., 1999; Rubin, 2002); interpret magma movement in volcanoes (Frèmont and Malone, 1987); and monitor pumping-induced seismicity (Lees, 1998; Ake et al., 2005).

In traditional approaches to absolute and relative location only early onset body waves, typically $P$ and/or $S$ waves, are used. The data utilized may be the direct arrival times; arrival-time difference computed between picked arrivals of two waveforms; or arrival-time differences inferred from time-lagged cross correlation of relatively small windows around the body-wave arrivals. In all three cases, the majority of the waveform is discarded. Furthermore, obtaining high accuracy with these techniques requires multiple stations with good azimuthal coverage. In this paper we demonstrate that it is possible to significantly reduce location uncertainty when few stations are available by using more of the waveform.

Coda refers to later arriving waves in the seismogram that arise from scattering (Aki, 1969; Snieder, 1999, 2006). Coda waves are ignored in most seismological applications due to the complexity involved in constraining complex hetergeneous velocity models in real settings. In this paper we develop an approach for locating earthquakes using coda waves. Snieder and Vrijlandt (2005) demonstrate that the coda of two earthquakes can be used to estimate the separation between them. Their technique, known as coda-wave interferometry (CWI), is based on the interference pattern between the coda waves. Unlike arrival-time-based location techniques, CWI does not require multiple stations or good azimuthal coverage. In fact, it is possible to obtain estimates of separation using a single station (Robinson, Sambridge, and Snieder, 2007). This makes CWI particularly interesting for regions where station density is low such as intraplate settings. In this paper we demonstrate how CWI separation estimates can be used to constrain location with data from a single station. Our technique can be used on coda waves alone or in combination with arrival times. We begin by introducing the theory of CWI-based earthquake location. This is followed by a demonstration of capability using synthetic examples and application to earthquakes on the Calaveras fault, California, using CWI alone and CWI in combination with arrival-time constraints.

\section{Theory}

Snieder and Vrijlandt (2005) introduce a CWI-based estimator of source separation $\delta_{\mathrm{CWI}}$ between two earthquakes,

$$
\delta_{\mathrm{CWI}}^{2}=g(\alpha, \beta) \sigma_{\tau}^{2},
$$

in which $\sigma_{\tau}$ is the standard deviation of the arrival-time perturbation between the coda waves of two earthquakes and $\alpha$ and $\beta$ are the near-source $P$ - and $S$ - wave velocities, respectively. The function $g$ depends on the type of excitation (explosion, point force, double couple) and on the direction of source displacement relative to the point force or double couple. For example, for two double-couple sources displaced in the fault plane

$$
g(\alpha, \beta)=7 \frac{\left(\frac{2}{\alpha^{6}}+\frac{3}{\beta^{6}}\right)}{\left(\frac{6}{\alpha^{8}}+\frac{7}{\beta^{8}}\right)},
$$

whereas, for two point sources in a $2 \mathrm{D}$ acoustic medium

$$
g(\alpha, \beta)=2 \alpha^{2}
$$

(Snieder and Vrijlandt, 2005). Snieder and Vrijlandt (2005) also show that for two double-couple sources that are not in the same fault plane

$$
\sigma_{\tau}^{2}=\frac{\left(\frac{6}{\alpha^{8}}+\frac{7}{\beta^{8}}\right) \delta_{\| \text {fault }}^{2}+2\left(\frac{1}{\alpha^{8}}+\frac{2}{\beta^{8}}\right) \delta_{\perp \text { fault }}^{2}}{7\left(\frac{2}{\alpha^{8}}+\frac{3}{\beta^{8}}\right)},
$$

in which $\delta_{\| \text {fault }}^{2}$ and $\delta_{\perp \text { fault }}^{2}$ are the separation parallel and perpendicular to the fault, respectively. In this paper we use equation (3) for the 2D examples. For the 3D examples, we use equation (2) which assumes that the source mechanism of both events are identical, an assumption likely to be true for events in the same fault plane. Robinson, Snieder, and Sambridge (2007) explore the impact of a change in mechanism.

The $\sigma_{\tau}$ in equation (1) is related to the maximum of the cross correlation between the coda of the two waveforms, $R_{\max }$, and hence can be computed directly from the recorded data. The original formulation by Snieder and Vrijlandt (2005) used a second-order Taylor series expansion of the waveform autocorrelation function to relate $\sigma_{\tau}$ and $R_{\max }$ by

$$
R_{\max }^{\left(t, t_{\mathrm{w}}\right)}=1-\frac{1}{2} \overline{\omega^{2}} \sigma_{\tau}^{2},
$$

in which $\overline{\omega^{2}}$ is the square of the dominant angular frequency

$$
\overline{\omega^{2}}=\frac{\int_{t-t_{\mathrm{w}}}^{t+t_{\mathrm{w}}} \dot{u}_{i}^{2}\left(t^{\prime}\right) d t^{\prime}}{\int_{t-t_{\mathrm{w}}}^{t+t_{\mathrm{w}}} u_{i}^{2}\left(t^{\prime}\right) d t^{\prime}},
$$

and $\dot{u}_{i}$ represents the time derivative of $u_{i}$. In this paper, we use the autocorrelation approach of Robinson et al. (2011) to relate the parameters directly, and we apply a restricted time lag search when evaluating $R_{\max }$. These extensions to the original technique of Snieder and Vrijlandt (2005) increase the range of applicability of CWI by 50\% (i.e., from 300 to $450 \mathrm{~m}$ separation for $1-5 \mathrm{~Hz}$ filtered coda waves.) 
Robinson et al. (2011) show that CWI leads to probabilistic constraints on source separation and introduce a Bayesian approach for describing the probability of true separation given the CWI data. Their approach is summarized by

$$
P\left(\tilde{\delta}_{\mathrm{t}} \mid \tilde{\delta}_{\mathrm{CWIN}}\right) \propto P\left(\tilde{\delta}_{\mathrm{CWIN}} \mid \tilde{\delta}_{\mathrm{t}}\right) \times P\left(\tilde{\delta}_{\mathrm{t}}\right)
$$

in which $P\left(\tilde{\delta}_{\mathrm{t}} \mid \tilde{\delta}_{\text {CWIN }}\right)$ is the posterior function indicating the probability of true separation $\tilde{\delta}_{\mathrm{t}}$ given the noisy CWI separation estimates $\tilde{\delta}_{\text {CWIN }} ; P\left(\tilde{\delta}_{\text {CWIN }} \mid \tilde{\delta}_{\mathrm{t}}\right)$ is the likelihood function (or forward model) giving the probability that the separation estimates $\tilde{\delta}_{\text {CWIN }}$ would be observed if the true separation was $\tilde{\delta}_{\mathrm{t}}$; and $P\left(\tilde{\delta}_{\mathrm{t}}\right)$ is the prior probability density function (PDF) accounting for all a priori information. The use of $N$ in $\delta_{\text {CWIN }}$ depicts CWI separations that include noise. The nomenclature is adopted here to remain consistent with Robinson et al. (2011) who study synthetically generated noise-free $\delta_{\mathrm{CWI}}$ and relate them to noisy estimates $\delta_{\mathrm{CWIN}}$. The tilde above the separation parameters in equation (7) indicates the use of a wavelength normalized separation parameter

$$
\tilde{\delta}=\frac{\delta}{\lambda_{\mathrm{d}}}
$$

which measures separation ( $\delta=\delta_{\mathrm{CWIN}}$ or $\delta_{\mathrm{t}}$ ) with respect to dominant wavelength $\lambda_{\mathrm{d}}$. In this paper we consider a uniform prior over appropriate bounds to ensure that the posterior function is dominated by the recorded data. The procedure for computing the likelihood $P\left(\tilde{\delta}_{\text {CWIN }} \mid \tilde{\delta}_{\mathrm{t}}\right)$ is derived by Robinson et al. (2011) and summarized in The Likelihood section in the Appendix. With these two pieces in place we can compute the posterior $P\left(\tilde{\delta}_{\mathrm{t}} \mid \tilde{\delta}_{\mathrm{CWIN}}\right)$ (or PDF) for the separation between any pair of events directly from their coda waves.

We seek a PDF which links individual pairwise posteriors $P\left(\tilde{\delta}_{\mathrm{t}} \mid \tilde{\delta}_{\mathrm{CWIN}}\right)$ to describe the location of multiple events whose maximum corresponds to the most probable combination of locations. More importantly, however, the PDF shall quantify location uncertainty and provide information on the degree to which individual events are constrained by the data. For convenience, we begin with three earthquakes having locations $\mathbf{e}_{1}, \mathbf{e}_{2}$, and $\mathbf{e}_{3}$. Using a Bayesian formulation we write

$$
P\left(\mathbf{e}_{1}, \mathbf{e}_{2}, \mathbf{e}_{3} \mid \mathbf{d}\right) \propto P\left(\mathbf{d} \mid \mathbf{e}_{1}, \mathbf{e}_{2}, \mathbf{e}_{3}\right) \times P\left(\mathbf{e}_{1}, \mathbf{e}_{2}, \mathbf{e}_{3}\right),
$$

in which $P\left(\mathbf{e}_{1}, \mathbf{e}_{2}, \mathbf{e}_{3} \mid \mathbf{d}\right), P\left(\mathbf{d} \mid \mathbf{e}_{1}, \mathbf{e}_{2}, \mathbf{e}_{3}\right)$, and $P\left(\mathbf{e}_{1}, \mathbf{e}_{2}, \mathbf{e}_{3}\right)$ are the posterior, likelihood, and prior functions, respectively. In equation (9), d represents observations that constrain the locations. They can be any combination of arrival times, geodetic information, or CWI separations. For example, if coda waves are used we have $P\left(\mathbf{e}_{1}, \mathbf{e}_{2}, \mathbf{e}_{3} \mid \tilde{\boldsymbol{\delta}}_{\text {CWIN }}\right)$ and $P\left(\tilde{\boldsymbol{\delta}}_{\text {CWIN }} \mid \mathbf{e}_{1}, \mathbf{e}_{2}, \mathbf{e}_{3}\right)$, in which $\tilde{\boldsymbol{\delta}}_{\text {CWIN }}$ are the wavelength normalized separation estimates. Alternatively, if we use CWI and arrival-time data we may write $P\left(\mathbf{e}_{1}, \mathbf{e}_{2}, \mathbf{e}_{3} \mid \tilde{\boldsymbol{\delta}}_{\mathrm{CWIN}}, \boldsymbol{\Delta}_{\mathrm{TT}}\right)$ and $P\left(\tilde{\boldsymbol{\delta}}_{\mathrm{CWIN}}, \boldsymbol{\Delta}_{\mathrm{TT}} \mid \mathbf{e}_{1}, \mathbf{e}_{2}, \mathbf{e}_{3}\right)$, in which $\boldsymbol{\Delta}_{\mathrm{TT}}$ represents the arrival-time differences. In the following derivation and in the Synthetic Experiments and Relocating Earthquakes on the Calaveras Fault sections we focus on the constraints imposed by coda waves, whereas in Combining Arrival-Time and CWI Constraints section we demonstrate how CWI and arrival-time data can be combined.

For three earthquakes we have likelihoods $P\left(\tilde{\delta}_{\mathrm{CWIN}, 12} \mid \mathbf{e}_{1}, \mathbf{e}_{2}\right), P\left(\tilde{\delta}_{\mathrm{CWIN}, 13} \mid \mathbf{e}_{1}, \mathbf{e}_{3}\right)$, and $P\left(\tilde{\delta}_{\mathrm{CWIN}, 23} \mid \mathbf{e}_{2}, \mathbf{e}_{3}\right)$. In writing these likelihoods we have replaced the conditional term on separation $\tilde{\delta}_{\mathrm{t}}$ with the locations (e.g., $\mathbf{e}_{1}$ and $\mathbf{e}_{2}$ ). This can be done because knowledge of location translates to separation. Note, however, that the reverse is not true. That is, knowledge of separation between a single event pair does not uniquely translate to location, but rather places a nonunique constraint on location. In other words, knowing $\left|\mathbf{e}_{1}-\mathbf{e}_{2}\right|$ and $\left|\mathbf{e}_{2}-\mathbf{e}_{3}\right|$ does not mean that $\left|\mathbf{e}_{1}-\mathbf{e}_{3}\right|$ is uniquely defined. Consequently, the likelihoods are weekly dependent, in that some likelihood-pairs share common events, an occurrence that becomes relatively less frequent as the number of events being located increases. For the purpose of this work we ignore this weak dependence and assume independence:

$$
\begin{aligned}
& P\left(\tilde{\delta}_{\mathrm{CWIN}} \mid \mathbf{e}_{1}, \mathbf{e}_{2}, \mathbf{e}_{3}\right) \approx P\left(\tilde{\delta}_{\mathrm{CWIN}, 12} \mid \mathbf{e}_{1}, \mathbf{e}_{2}\right) \\
& \quad \times P\left(\tilde{\delta}_{\mathrm{CWIN}, 13} \mid \mathbf{e}_{1}, \mathbf{e}_{3}\right) \times P\left(\tilde{\delta}_{\mathrm{CWIN}, 23} \mid \mathbf{e}_{2}, \mathbf{e}_{3}\right) .
\end{aligned}
$$

Similarly, the earthquake locations are independent and the joint prior becomes

$$
P\left(\mathbf{e}_{1}, \mathbf{e}_{2}, \mathbf{e}_{3}\right)=P\left(\mathbf{e}_{1}\right) \times P\left(\mathbf{e}_{2}\right) \times P\left(\mathbf{e}_{3}\right) .
$$

Combining equations (10) and (11) gives the joint posterior function

$$
\begin{aligned}
P\left(\mathbf{e}_{1}, \mathbf{e}_{2}, \mathbf{e}_{3} \mid \tilde{\boldsymbol{\delta}}_{\mathrm{CWIN}}\right)= & c \prod_{i=1}^{3} P\left(\mathbf{e}_{i}\right) \\
& \times \prod_{i=1}^{2} \prod_{j=i+1}^{3} P\left(\tilde{\delta}_{\mathrm{CWIN}, i j} \mid \mathbf{e}_{i}, \mathbf{e}_{j}\right)
\end{aligned}
$$

for three events.

A detailed understanding of the location of a single event (e.g., $\mathbf{e}_{2}$ ) is obtained by computing the marginal

$$
P\left(\mathbf{e}_{2} \mid \delta_{\mathrm{CWIN}}\right)=\iint P\left(\mathbf{e}_{1}, \mathbf{e}_{2}, \mathbf{e}_{3} \mid \tilde{\delta}_{\mathrm{CWIN}}\right) d \mathbf{e}_{1} d \mathbf{e}_{3},
$$

in which the intergral is taken over all plausible locations for $\mathbf{e}_{1}$ and $\mathbf{e}_{3}$. Alternatively, we can compute the marginal for a single event coordinate by integrating the posterior over all events and remaining coordinates for the chosen earthquake. Evaluation of the normalizing constant $c$ in equation (12) involves finding the integral of the posterior function over all plausible locations. In many applications the constant of proportionality $c$ can be ignored. For example, it is not required when seeking the combination of locations which 
maximize the posterior function, nor in Bayesian sampling algorithms such as Markov-chain Monte-Carlo techniques which only require evaluation of a function proportional to the PDF.

Extending to $n$ events we get the posterior function

$$
\begin{aligned}
P\left(\mathbf{e}_{1}, \ldots, \mathbf{e}_{n} \mid \tilde{\delta}_{\mathrm{CWIN}}\right)= & c \prod_{i=1}^{n} P\left(\mathbf{e}_{i}\right) \\
& \times \prod_{i=1}^{n-1} \prod_{j=i+1}^{n} P\left(\tilde{\delta}_{\mathrm{CWIN}, i j} \mid \mathbf{e}_{i}, \mathbf{e}_{j}\right) .
\end{aligned}
$$

When evaluating equation (14) over a range of locations it is necessary to compute and multiply many numbers close to zero. This is because the PDFs tend to zero as the locations get less likely (i.e., near the boundaries of the plausible region). Such calculations are prone to truncation errors, so we work with the negative logarithm

$$
L\left(\mathbf{e}_{1}, \mathbf{e}_{2}, \ldots, \mathbf{e}_{n}\right)=-\ln \left[P\left(\mathbf{e}_{1}, \ldots, \mathbf{e}_{n} \mid \tilde{\delta}_{\mathrm{CWIN}}\right)\right]
$$

or

$$
\begin{aligned}
L\left(\mathbf{e}_{1}, \mathbf{e}_{2}, \ldots, \mathbf{e}_{n}\right)= & -\ln [c]-\sum_{i=1}^{n} \ln \left[P\left(\mathbf{e}_{i}\right)\right] \\
& -\sum_{i=1}^{n-1} \sum_{j=i+1}^{n} \ln \left[P\left(\tilde{\delta}_{\mathrm{CWIN}, i j} \mid \mathbf{e}_{i}, \mathbf{e}_{j}\right)\right] .
\end{aligned}
$$

The logarithm improves numerical stability by replacing products with summations. The negative facilitates the use of optimization algorithms that are designed to minimize an objective function.

The event locations $\mathbf{e}_{1}, \mathbf{e}_{2}, \ldots, \mathbf{e}_{n}$ are defined by coordinates $\hat{x}, \hat{y}$, and $\hat{z}$, in which the hat indicates use of a local coordinate system. We choose a local coordinate system which removes ambiguity associated with transformations of the coordinate system. It is necessary to do this because the distances between events are invariant for rotations, reflections, and translations of the seismicity pattern, hence cannot be resolved from CWI alone. In defining this coordinate system we fix the first event at the origin

$$
\mathbf{e}_{1}=(0,0,0),
$$

the second event on the positive $\hat{x}$ axis

$$
\mathbf{e}_{2}=\left(\hat{x}_{2}, 0,0\right), \quad \hat{x}_{2}>0,
$$

the third on the $\hat{x}-\hat{y}$ plane

$$
\mathbf{e}_{3}=\left(\hat{x}_{3}, \hat{y}_{3}, 0\right), \quad \hat{y}_{3}>0,
$$

and the fourth to

$$
\mathbf{e}_{4}=\left(\hat{x}_{4}, \hat{y}_{4}, \hat{z}_{4}\right), \quad \hat{z}_{4}>0 .
$$

This coordinate system reduces translational (equation 17) and rotational (equations 18-20) nonuniqueness without loss of generality. It is necessary to work with a local coordinate system when using coda waves alone because the CWI technique constrains only event separation between earthquakes. The inclusion of arrival times in the Combining Arrival-Time and CWI Constraints section allows us to move to a global reference system.

In summary, the posterior $P\left(\mathbf{e}_{1}, \ldots, \mathbf{e}_{n} \mid \tilde{\delta}_{\text {CWIN }}\right)$ and its negative logarithm $L$ describe the joint probability of multiple event locations given the observed coda waves. The most likely set of locations is given by the minimum of $L$. In this paper we use the Polak-Ribiere technique (Press et al., 1987), a conjugate gradient method, to minimize $L$. It uses the derivatives of $L$, derived in the Derivatives section of the Appendix, to guide the optimization procedure. Note that when optimizing equation (16) the values of $\ln [c]$ and $\ln \left[P\left(e_{i}\right)\right]$ can be ignored because they are constant $\left(\ln \left[P\left(e_{i}\right)\right]\right.$ is constant because we consider a uniform prior).

\section{Synthetic Experiments}

We use synthetic examples in 2D and 3D with 50 earthquakes to test the performance of the optimization routine. In these examples the synthetic earthquakes are located randomly and CWI data generated according to the event separation. It is not necessary to generate synthetic waveforms and compute CWI estimates directly because we are testing the performance of the optimization routine only. The ability of CWI to estimate event separation has been demonstrated already (Snieder and Vrijlandt, 2005; Robinson, Sambridge, and Snieder, 2007; Robinson et al., 2011). We undertake a complete coda-wave location experiment, including the calculation of CWI separation estimates, for recorded earthquakes in Relocating Earthquakes on the Calaveras Fault and in Combining Arrival-Time and CWI Constraints sections.

\section{Examples 1 and 2: 2D Synthetic Experiments}

We design a 2D synthetic acoustic experiment (example 1) to test the performance of our CWI-based relative location algorithm by randomly selecting $\hat{x}$ and $\hat{y}$ coordinates such that $-50 \leq \hat{x}, \hat{y} \leq 50 \mathrm{~m}$. These are indicated with triangles in Figure 1 . We assume a local velocity of $\alpha=3300 \mathrm{~m} / \mathrm{s}^{-1}$ between all event pairs and a dominant frequency of $2.5 \mathrm{~Hz}$ to represent waveform data filtered between 1 and $5 \mathrm{~Hz}$. The purpose of these examples is to synthetically test the location algorithm. Hence, we do not need to synthetically generate waveforms and compute CWI separation estimates, Rather, we begin by synthetically generating the CWI separation estimates directly. Robinson et al. (2011) showed that the CWI data are defined by the dominant wavelength normalized positive bounded Gaussian PDF with statistics $\bar{\mu}_{\mathrm{N}}$ and $\bar{\sigma}_{\mathrm{N}}$. A hypothetical CWI mean is created by setting

$$
\bar{\mu}_{\mathrm{N}}=\mu_{1}\left(\tilde{\delta}_{t}\right),
$$

using equation (A7). This assumption ensures that the sample mean of hypothetical separation estimates is consistent with known CWI biases (Robinson et al., 2011). In example 


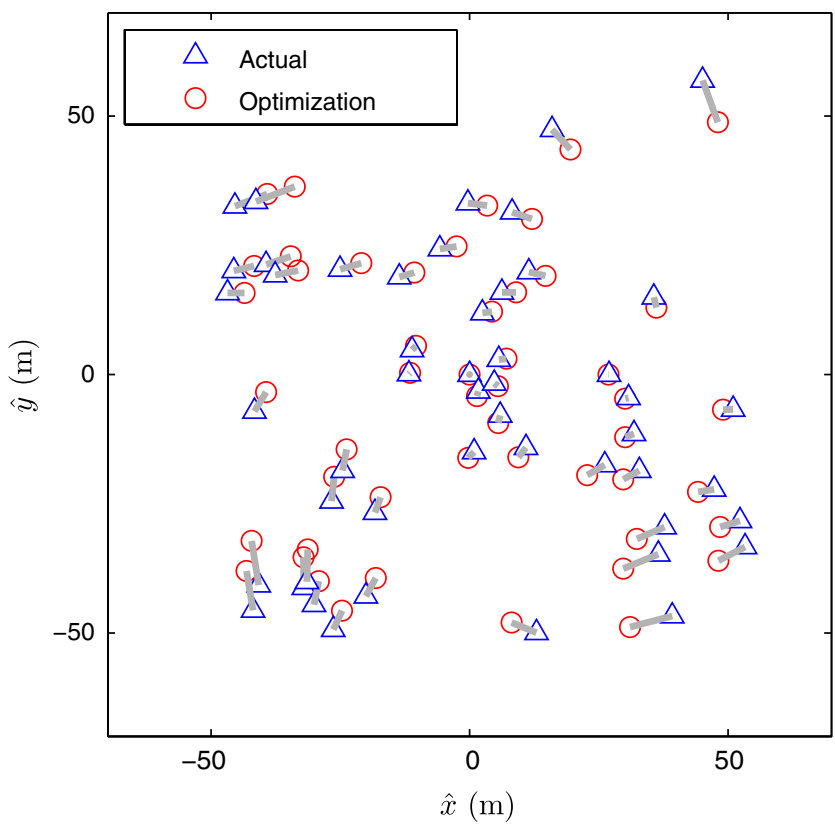

Figure 1. Example 1: Synthetic relocation of 50 earthquakes in $2 \mathrm{D}$ using all constraints with noise $\bar{\sigma}_{\mathrm{N}}=0.02$. Actual and optimization event locations are identified by triangles and circles, respectively. The color version of this figure is available only in the electronic edition.

1 we use $\bar{\sigma}_{\mathrm{N}}=0.02$ between all event pairs. Application of our optimization procedure on the hypothetical CWI data yields the circles in Figure 1. The optimization does not lead to the exact solution due to the addition of noise $\left(\bar{\sigma}_{\mathrm{N}}=0.02\right)$ on the hypothetical CWI data. The average coordinate error is $2.0 \mathrm{~m}$ (average location error $\approx 4 \mathrm{~m}$ ); this is small compared to the noise of $\bar{\sigma}_{\mathrm{N}}=0.02$, which for $V_{S}=3300 \mathrm{~m} / \mathrm{s}^{-1}$ and $f_{\text {dom }}=2.5 \mathrm{~Hz}$ corresponds to roughly $25 \mathrm{~m}$.

Robinson et al. (2011) demonstrates the noise on CWI estimates is often larger than 0.02 and it increases with event separation. Consequently, example 1 is simplistic because we fix $\bar{\sigma}_{\mathrm{N}}=0.02$ for all pairs. In example 2 we increase the uncertainty and introduce a distance dependence into the hypothetical $\bar{\sigma}_{\mathrm{N}}$ by defining $\bar{\sigma}_{\mathrm{N}}=\epsilon\left(\delta_{\mathrm{t}}\right)$, in which $\epsilon\left(\delta_{\mathrm{t}}\right)$ is the half-width of the error bars for a synthetic acoustic experiment with filtering between 1 and $5 \mathrm{~Hz}$ (see fig. 4b of Robinson et al., 2011). Repeating the optimization leads to the circles in Figure 2 which have an average coordinate error of $2.8 \mathrm{~m}$ (average location error $\approx 9 \mathrm{~m}$ ).

Conjugate gradient-based optimization techniques are susceptible to the presence of local minima. This is because they use the slope of the target function to explore the solution space. We explore the impact of local minima for our CWI location problem by beginning the optimization from 25 randomly chosen starting positions. We observe negligible difference in the solutions indicating that neither example is susceptible to local minima.

Three observations can be drawn from the error structure in Figures 1 and 2. First, the location errors depicted by gray bars increase between examples 1 and 2 with the introduction

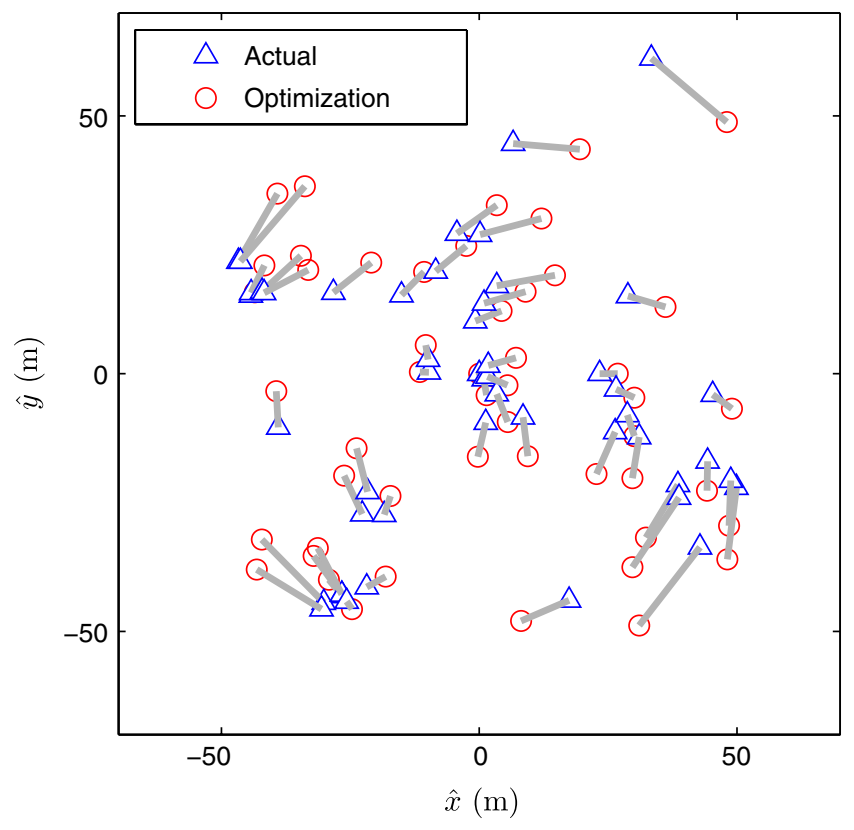

Figure 2. Example 2: Synthetic relocation of 50 earthquakes in $2 \mathrm{D}$ using all constraints with noise $\bar{\sigma}_{\mathrm{N}}=2 \epsilon\left(\delta_{t}\right)$. Actual and optimization event locations are identified by triangles and circles, respectively. The color version of this figure is available only in the electronic edition.

of larger noise. Second, the errors are larger for events at greater distances from the center. This is because events near the center of the cluster are constrained by links from all angles, whereas those on the outside are moderated by links from a limited number of directions. This observation is analogous to problems associated with poor azimuthal coverage in triangulation such as individual earthquake location from limited arrival-time data or Global Positioning System positioning with few satellites. Third, the location errors form a pattern of circular rotation, despite our attempt to correct for rotational nonuniqueness with the local coordinate system.

The local coordinate system works by constraining the location of the first three earthquakes. Earthquake 1 is fixed at the origin, earthquake 2 on the positive $\hat{x}$ axis, and earthquake 3 has $\hat{y}>0$. As the number of events increase the strength of these constraints on later events weakens allowing small rotations of events with respect to each other. That is, even though the rotational freedom of the cluster is in principle removed by the constraints imposed on the events (see equations 17-19; equation 20 is needed in 3D only) we observe that in practice the presence of noise allows the rotational nonuniqueness to reappear. This is because errors align themselves in directions least constrained by data. For the CWI technique this amounts to rotations in 2D. The same phenomenon is observed in linear inversion where noise creates large spurious model changes in directions of the eigenvectors with the smallest singular values (Aster et al., 2005). Fortunately, however, combining coda waves with measurements of arrival times alleviates this problem and 
facilitates the removal of a local coordinate system altogether (see the Combining Arrival-Time and CWI Constraints section). On balance, however, we gain confidence in the optimization procedure due to its stability for different starting locations and because of the small average coordinate errors of 2.0 and $2.8 \mathrm{~m}$ for examples 1 and 2, respectively.

Example 3: The Impact of Incomplete Event

Pairs in 2D

Synthetic examples 1 and 2 use 100\% direct linkage between event pairs. That is, there is a constraint between each earthquake and all other events. In reality, we might expect that the separation between some pairs will not be constrained by CWI data due to poor signal-to-noise ratio in the coda for common stations. Obviously, the fewer stations that record an event the more likely it is that links between it and other events will be broken. In such cases the probabilistic distance constraint between a pair of events may only exist indirectly through multiple pairs. In this section we consider the impact of reduced linkage between event pairs. In example 3, we repeat example 2 using $90 \%, 80 \%, \ldots, 10 \%$ of the links. That is, we randomly select $10 \%$ of the event pairs and remove the separation estimates between those pairs to create a data set with $90 \%$ linkage. Then, we randomly remove $20 \%$ of the links and so on. This experiment is designed to mimic a realistic recording situation in which CWI estimates are not available for all event pairs due to station problems, poor signal-to-noise ratio or any number of other reasons. As with the above examples, we undertake the optimization with 25 randomly chosen starting locations.

Figure 3 illustrates the maximum $\Delta_{\max }$ (top) and mean $\Delta_{\mu}$ (middle) of the coordinate error as a function of percentage of earthquake pairs that are directly linked by a separation estimate. We show the statistics for the best optimization solution (thick) and for the solution space when all 25 optimizations are considered (thin). In the former case the best solution is determined by the set of event locations which lead to the smallest value of $L$. The error in the best solution is consistent when $30 \%$ or more of the branches are used. The errors increase when only $10 \%$ or $20 \%$ of the constraints are included. Interestingly, this breakdown around $20 \%-30 \%$ coincides with the point where the average number of branches required to link an event pair reaches 2 (see Fig. 3, bottom). Because the average number of branches can be computed in advance it can be used as an indication of inversion stability prior to optimization. A higher breakdown is observed when all 25 solutions are considered collectively. For example, the maximum coordinate error $\Delta_{\max }$ exceeds that for the best solution for linkage $\leq 60 \%$ confirming that the optimization is susceptible to local minima and that a range of starting points should be considered. Some optimizations fail to converge after 1200 iterations when the linkage is $60 \%$ or lower. All optimizations fail when the linkage is $20 \%$ or lower.
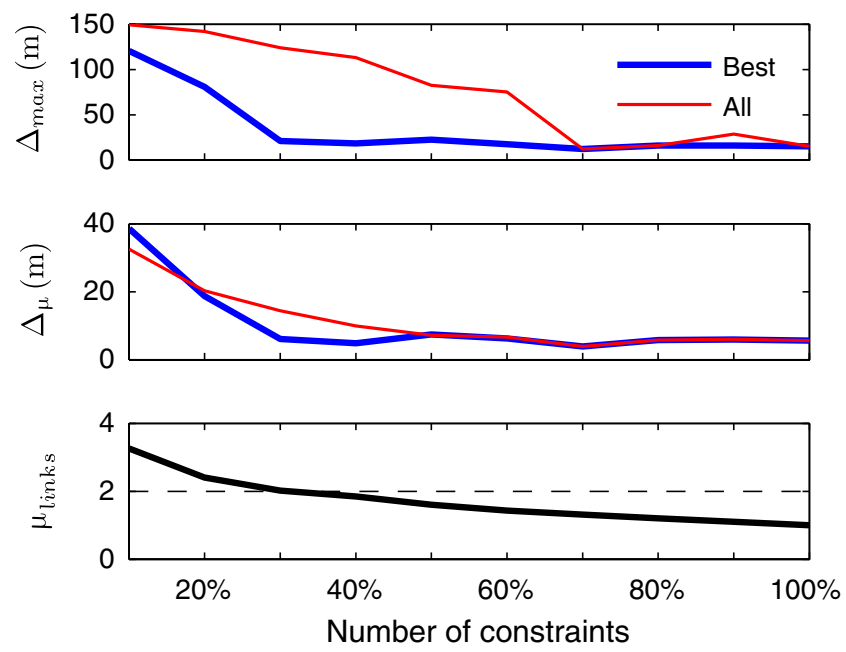

Figure 3. Example 3: Statistical measures of error in the solutions for the 2D synthetic cases when all and best optimization results are considered. The statistics $\Delta_{\max }$ and $\Delta_{\mu}$ are the maximum and mean coordinate error, respectively. The bottom subplot shows the average minimum number of branches required to link the 2450 pairs. The color version of this figure is available only in the electronic edition.

The derivatives used in the conjugate gradient method depend on events connected by CWI measurements. Consequently, earthquakes that are only connected via other events do not communicate with each other directly. To some extent, this should be addressed during the iterative process where location information can spread to events which have no direct links. However, the lack of direct connection through the gradient could prevent convergence in extreme cases or, more likely, slow the procedure down. This could explain why some examples do not converge after 1200 iterations. VanDecar and Snieder (1994) show that derivative-based regularization acts slowly through iterative least squares, because every cell in one iteration communicates only with its neighbors, and they demonstrate that this can be fixed with preconditioning in some cases. Their findings suggest that it may be possible to improve the convergence (stability and/or speed) of the CWI optimization by preconditioning.

Example 4: The Impact of Incomplete Event

Pairs in 3D

In example 4 we expand the optimization routine to 3D by randomly picking a set of event locations for 50 earthquakes with $-50 \mathrm{~m} \leq \hat{x}, \hat{y}, \hat{z} \leq 50 \mathrm{~m}$. As in the $2 \mathrm{D}$ case we assume a local velocity of $v=3300 \mathrm{~m} / \mathrm{s}^{-1}$ between all event pairs and a dominant frequency of $2.5 \mathrm{~Hz}$ to represent waveform data filtered between 1 and $5 \mathrm{~Hz}$. The hypothetical CWI mean is created using equation (21) which ensures consistency between the sample mean of hypothetical separation estimates and CWI biases. We use a standard deviation for the noisy CWI estimates of $\bar{\sigma}_{\mathrm{N}}=\epsilon\left(\delta_{\mathrm{t}}\right)$, in which $\epsilon\left(\delta_{\mathrm{t}}\right)$ is the same as that used in examples 2 and 3 , and perform the 

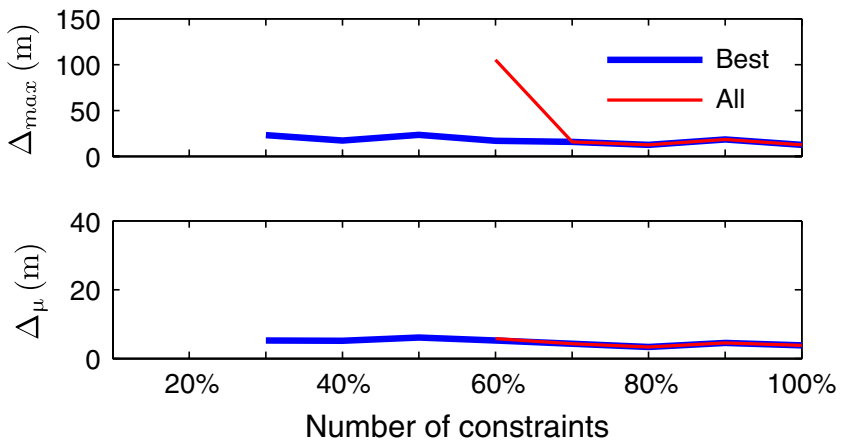

Figure 4. Example 4: Statistical measures of error in the optimization solutions for the 3D synthetic cases when all and best results are considered. The statistics $\Delta_{\max }$ and $\Delta_{\mu}$ are the maximum and mean coordinate error, respectively. The absence of the lines below $60 \%$ and $30 \%$ indicates a breakdown in the solutions when all or best optimization result(s) are considered, respectively. The color version of this figure is available only in the electronic edition.

optimization using $10 \%, 20 \%, \ldots, 100 \%$ of the direct links. In each case we repeat the optimization 25 times using randomly chosen starting locations. The results are summarized in Figure 4.

When $70 \%$ of the direct constraints are considered all optimization results (thin) are consistent with the best solution obtained from all 25 starting locations (thick). The best solution constrains the event locations down to $30 \%$ of the direct links. This is depicted in Figure 4 by the absence of the thin lines below $60 \%$ and thick lines below $30 \%$ linkages. The accurate convergence of the best solution for cases with $30 \%$ linkage or higher is encouraging for the potential of coda-wave optimization to constrain earthquake location.

\section{Summary of Synthetic Experiments}

In summary, the synthetic examples demonstrate the ability of coda-wave data to constrain relative event location using optimization. The optimization error is rotational in nature and influenced by the noise on CWI estimates with greater $\bar{\sigma}_{\mathrm{N}}$ leading to larger errors in the solutions. In 3D, when $70 \%$ or more of the direct branches are used the optimizer is stable with no observable difference in the solution for 25 randomly chosen starting locations. As the direct linkage reduces to $50 \%$ the optimization becomes less stable and the best solution from 25 random starting locations is required to find the optimal solution. All optimizations fail to converge as the number of links decrease below $30 \%$.

\section{Relocating Earthquakes on the Calaveras Fault}

In this section we relocate 68 earthquakes from the $\mathrm{Cal}-$ averas fault, California. The 68 earthquakes are selected from the 308 earthquake Calaveras examples released with the open source double-difference algorithm, or hypoDD (Waldhauser and Ellsworth, 2000; Waldhauser, 2001; see also Data and Resources). These events are chosen for four reasons. First, they are recorded by a large number of stations (Fig. 5)

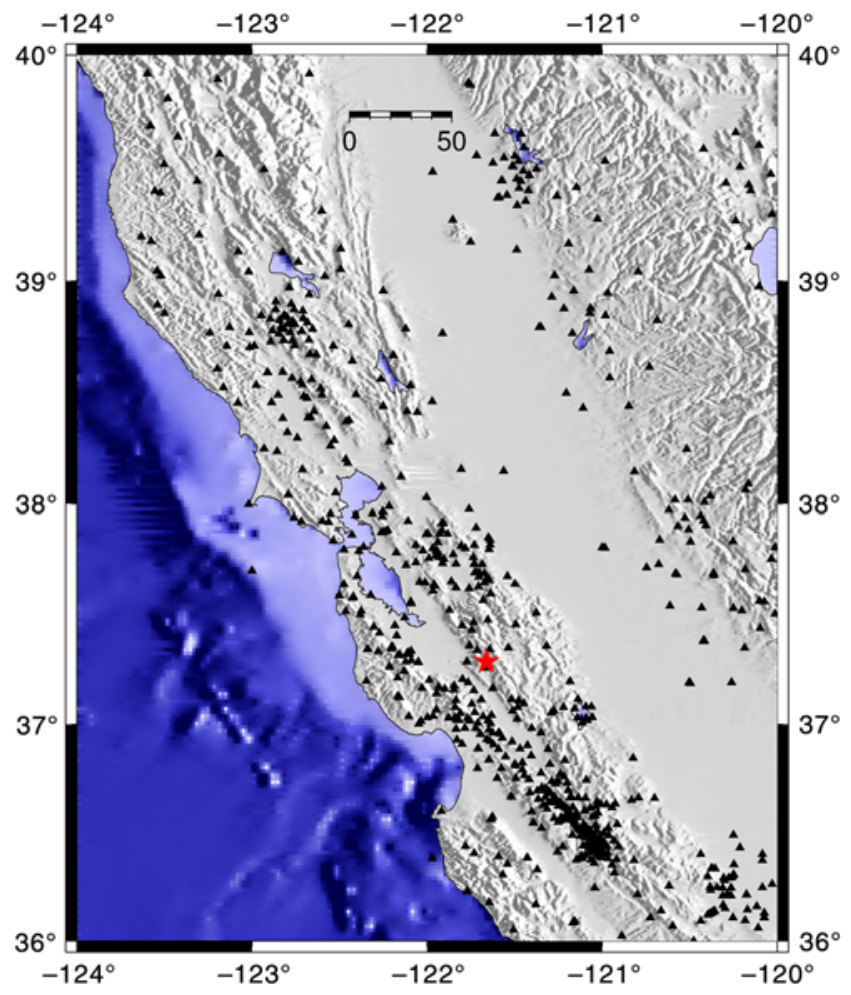

Figure 5. Location of the Calaveras cluster (star) and 805 seismic stations (triangles). The color version of this figure is available only in the electronic edition.

and therefore lend themselves to accurate arrival-time location. This makes them ideal for assessing the performance of a new location technique. Second, they are distributed with separations from near zero to hundreds of meters making them ideal for application of CWI. Third, Calaveras earthquakes have been well researched with several studies having relocated events in the region (Waldhauser, 2001; Schaff et al., 2002; Waldhauser and Schaff, 2008). Finally, the hypoDD locations for these 68 earthquakes align in a streak increasing the likelihood that they have near identical source mechanisms, a necessary assumption for the application of equation (2). The relocations in this paper are sorted into four examples as summarized in Table 1. Waveforms, cross correlations and separation estimates for six Calaveras event

Table 1

Location Examples for the 68 Calaveras Earthquakes

\begin{tabular}{cc}
\hline Example & \multicolumn{1}{c}{ Description } \\
\hline 5 & $\begin{array}{c}\text { Comparison of CWI, catalog, and hypoDD locations } \\
\text { (using all available data). } \\
\text { Exploration of station dependence for CWI and } \\
\text { hypoDD (using a subset of data). } \\
\text { Combined use of CWI and arrival-time data with all } \\
\text { and a reduced number of stations. } \\
\text { Combined use of CWI and arrival-time data when } \\
\text { arrival times constrain only 50\% of the events. }\end{array}$ \\
\hline
\end{tabular}




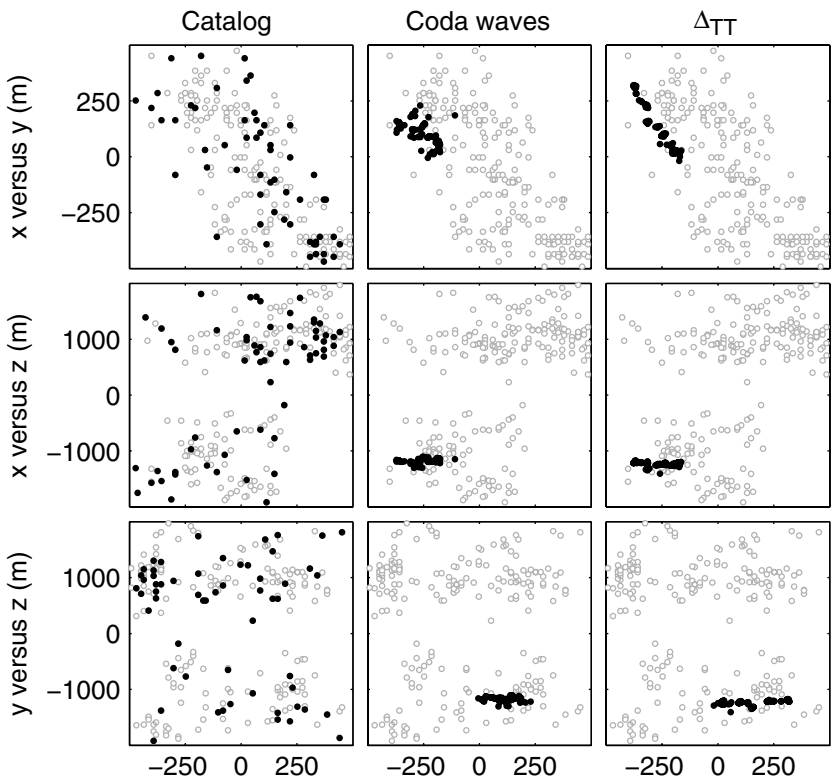

Figure 6. Example 5: Comparison of relative earthquake locations using three different methods: catalog location (column 1), CWI (column 2), and hypoDD (column 3). Note that in the case of the hypoDD and CWI inversions, we consider only the 68 earthquakes in black; the gray catalog locations for the remaining 240 earthquakes are shown for the purpose of orientation only. In this and subsequent similar figures (Figs. 8, 9, 11, and 13), $x$ is defined as positive toward the east, $y$ is positive toward the north, and $z$ is positive down.

pairs are illustrated by Robinson et al. (2011) and are not repeated here for the sake of brevity.

\section{Example 5: Comparison of CWI, Catalog and HypoDD Locations}

Figure 6 illustrates three sets of locations for the Calaveras earthquakes. The first column shows the original cata$\log$ locations for all 308 earthquakes. That is, each event is located individually using all available arrival-time data and a regional velocity model. The 68 earthquakes of interest in this study are differentiated in black. Catalog locations suggest that the 68 earthquakes of interest are spatially widely distributed on the scale of Figure 6.

To apply CWI we download available waveforms from the Northern California Earthquake Data Center (see Data and Resources). Unsuitable waveforms are removed using the conditions summarized in Table 2. Remaining waveforms are filtered between 1 and $5 \mathrm{~Hz}$ and aligned to $P$ arrivals at $0 \mathrm{~s}$. CWI estimates are obtained from $5 \mathrm{~s}$ wide nonoverlapping time windows between $2.5<t \leq 20 \mathrm{~s}$ and used to create probabilistic constraints on event separation. We utilize the local coordinate system introduced in the Theory section and find the optimum relative locations using Polak-Ribiere optimization.

In this, and the following Calaveras examples, we allow the earthquakes to move freely in all three directions during the inversion despite using the in-fault separation estimates
Table 2

Conditions Used to Identify Unsuitable Waveforms before Applying CWI

Condition

\begin{tabular}{ll}
\hline \multicolumn{1}{c}{ Condition } \\
\hline 1 & Waveform is clearly corrupted. \\
2 & Waveform indicates recording of more then one event. \\
3 & Signal-to-noise ratio is obviously low. \\
4 & There is insufficient coda recorded after the first arrivals. \\
5 & There is insufficient recording before the arrivals (needed for \\
& accurate noise energy estimate). \\
\hline Originally published as table 5 in Robinson et al. (2011).
\end{tabular}

Originally published as table 5 in Robinson et al. (2011).

given by equation (2). We allow the events to move freely so that we can test the performance of our algorithm without assuming a priori that the earthquakes are constrained on the same fault plane. We approximate the true event separation using the in-fault separation of equation (2) so that we can focus on developing a working algorithm and demonstrate capability without dealing with the complexity of in-fault $\left(\delta_{\| \text {fault }}\right)$ and out-of-fault $\left(\delta_{\perp \text { fault }}^{2}\right)$ displacement. Considering the more complicated formulation of equation (4) is left for future work. Another potential focus for future work involves refining our algorithm to simultaneously resolve event location and representative fault plane by restricting the events to align in a single (unknown a priori) plane. That is, for cases where the earthquakes are believed $a$ priori to be in the same plan.

CWI locations for the 68 events are illustrated in column two of Figure 6. Catalog locations (gray) are shown for the remaining 240 earthquakes and are included to ease comparison. The third column of Figure 6 illustrates the locations given by hypoDD with singular value decomposition (SVD), absolute arrival times and cross-correlation-computed arrival-time differences.

Absolute locations cannot be found by CWI alone. This is because of the nonuniqueness associated with translation, rotation, and reflection. For the sake of comparison, we arbitrarily choose a master event and translate our relative locations to align with the hypoDD location for that event. This arbitrary translation does not change the relative locations. We return to this issue of relative versus absolute location in example 7 by introducing a combined arrival-time and coda-wave inversion.

The spatial distribution of the CWI locations is clearly tighter than the catalog locations of column 1 . That is, CWI provides an independent indication of clustering for the 68 events and to first order, similar locations to those from hypoDD (column 3). There is a small second-order difference between the CWI- and hypoDD-based locations. In particular, the lineation is less clear in the CWI locations (column 2) than the hypoDD locations (column 3). Our experience suggests that the CWI locations are less supportive of the presence of streaks although a complete understanding of these differences is left for future work. Our attention now is devoted toward understanding how both techniques perform 


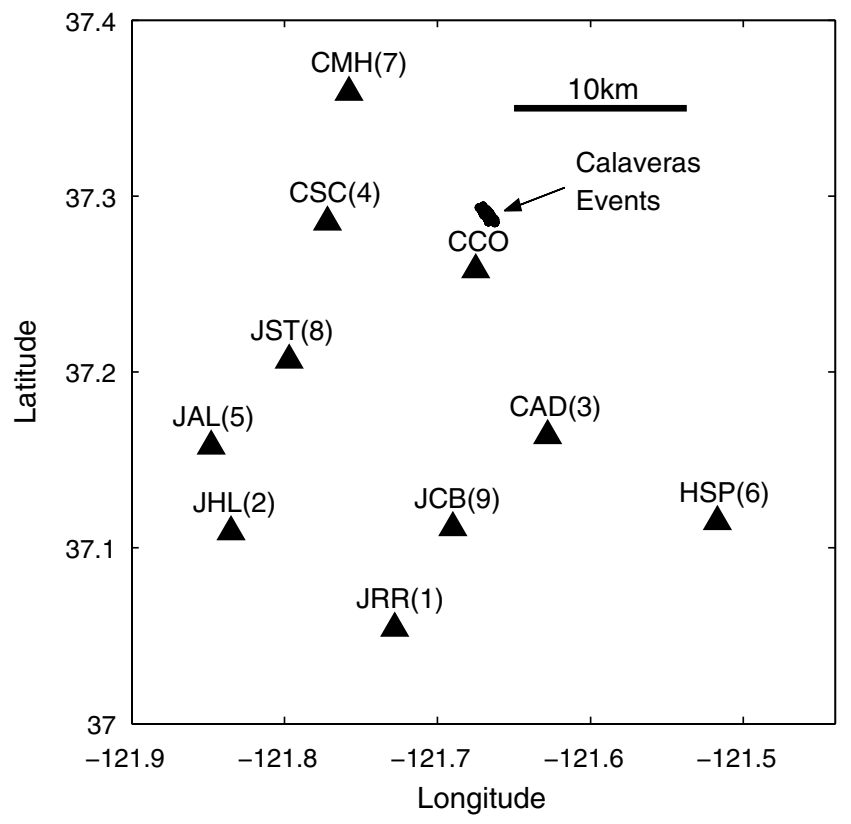

Figure 7. Location of the 10 stations (triangles) used to relocate the Calaveras events in examples 6-8. Stations are removed one at a time, according to the order defined by the numbers in parentheses. That is, JRR is the first station to be removed, JHL is the second, and so on. Events are indicated with circles.

with fewer stations (example 6) and exploring how CWI and arrival times can be combined (examples 7 and 8).

\section{Example 6: Dependence on the Number of Stations}

Accurate location of the Calaveras events is possible using arrival phases because of the excellent recording situation in California with many stations and strong azimuthal coverage (see Fig. 5). In contrast, a small number of stations and poor azimuthal coverage are common limitations when trying to locate intraplate clusters. For example, there are only four network seismic stations in the South West Seismic Zone of western Australia, a region similar in size to that hosting 805 stations in Figure 5.

We explore the impact of poorer recording situations in example 6 by relocating the 68 Calaveras events using hypoDD and coda waves with a reduced number of stations. We begin with 10 stations and repeat the process removing one at a time until a single station remains. The 10 stations and their order of removal are shown in Figure 7.

CWI locations are illustrated in Figure 8 for the inversions with seven, five, four, three, two, and one station. We observe a high level of consistency between these six inversions and the locations shown in Figure 6 (column 2) when all stations are considered. That is, the coda-wave approach is self-consistent regardless of the number of stations available, reinforcing our claim that coda waves can constrain location in what would normally be regarded as a poor station network.

Figure 9 illustrates the hypoDD inversion results for seven, five, and four stations. The arrival-time problem is ill-posed for fewer than four stations so it is not possible to apply hypoDD with SVD for three or fewer stations. The hypoDD locations are less self-consistent as the number of stations is reduced. We observe a general increase in scatter and a higher number of stray events outside the cluster when less stations are used with hypoDD. Even with seven stations the linear geometry of Figure 6 (column 3) is less evident.
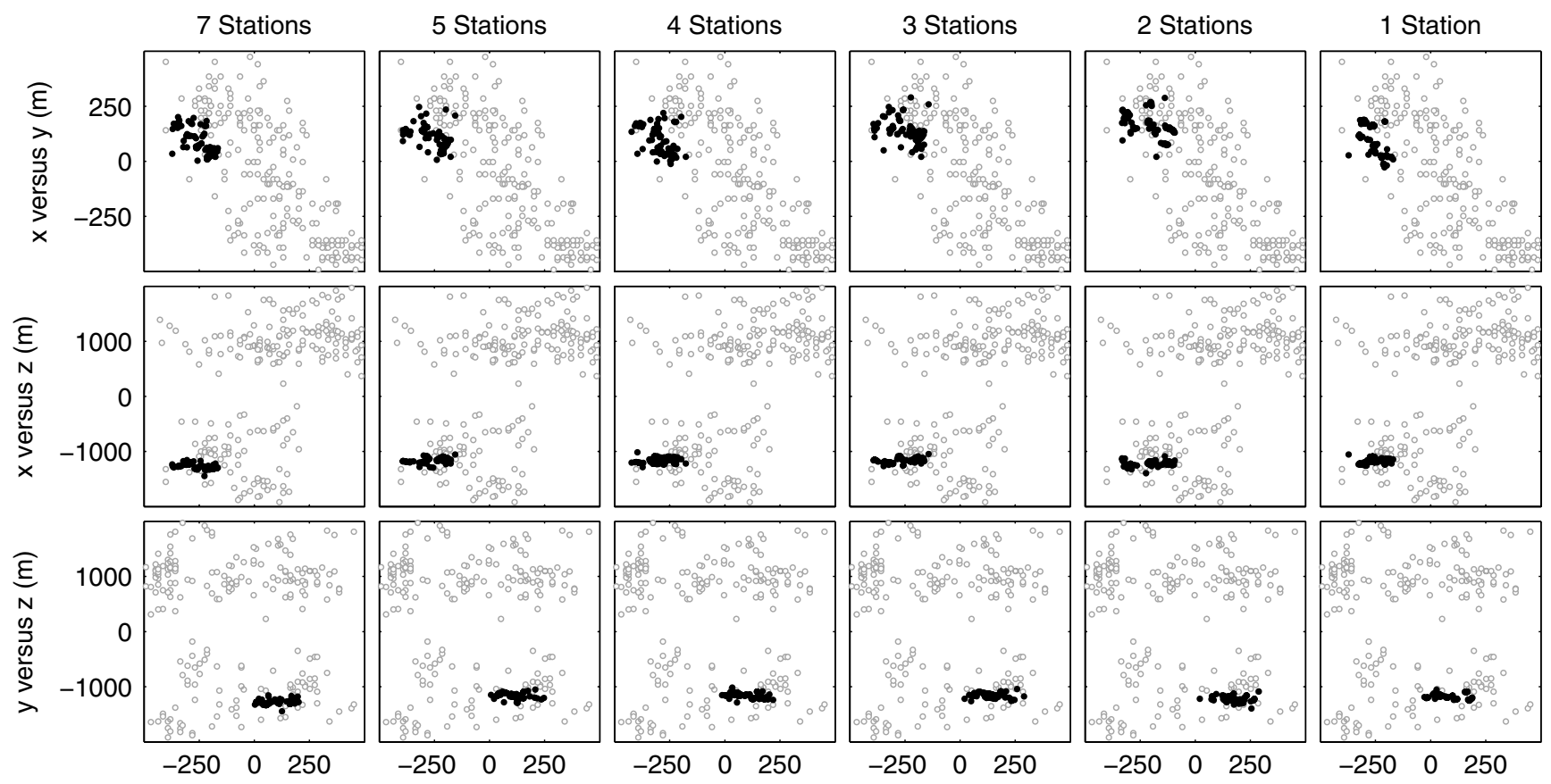

Figure 8. Example 6: CWI relative locations with reduced stations. Axes as defined in Figure 6. 

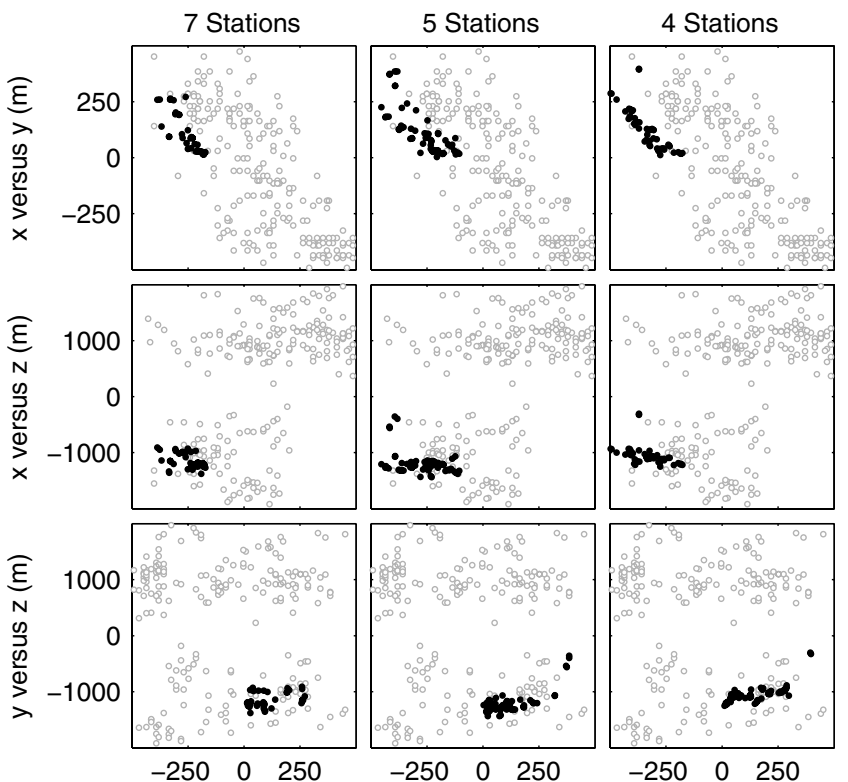

Figure 9. Example 6: HypoDD (SVD) relative locations with reduced stations. Axes as defined in Figure 6.

As the number of stations is reduced neither the CWI nor hypoDD techniques are able to relocate all events. To use the coda waves we need at least one pairwise separation constraint to be formed from the available stations. This means that for every event there must be at least one station that records it, and at least one other earthquake, sufficiently well to apply CWI. Fortunately, we can make an assessment of this prior to starting the inversion. The top panel of Figure 10 demonstrates that when five or more stations are used, CWI can constrain the location of all 68 earthquakes. When less than five stations are used the coda waves constrain a decreasing number of events until at one station it is only possible to locate 55 of the 68 events. The hypoDD algorithm also fails to locate all events as the number of stations is reduced. In the case of hypoDD an event can be identified as unconstrainable in one of two stages. First, the data are analyzed to ensure that there exist arrival-time differences for each event and at least one other earthquake. This is analogous to the situation for the coda-wave technique. The hypoDD program also has a secondary identification phase in which events that can not be located sufficiently well are rejected during the inversion. This process is related to the iterative removal of outliers described by Waldhauser and Ellsworth (2000). The top panel of Figure 10 shows that the number of events relocated by hypoDD fluctuates between 63 and 28 earthquakes for 10 to 4 stations, and it demonstrates that the number of events located by hypoDD is less than or equal to the number located by CWI.

The remaining panels of Figure 10 illustrate a statistical comparison of the CWI and hypoDD locations with a reduced number of stations to those using hypoDD with all available data. For the CWI inversions, the mean and maximum coordinate difference is consistent regardless of the number of
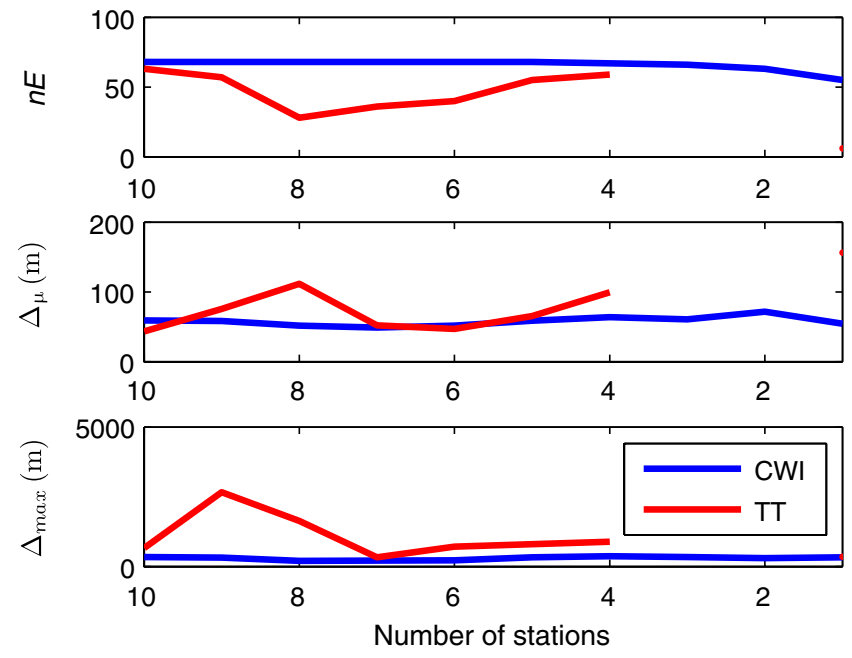

Figure 10. Example 6: Number of constrainable events $n E$ in the CWI and hypoDD inversions as a function of the number of stations considered (top). Mean (middle) and maximum (bottom) of the difference computed between the reduced station inversion results (CWI and hypoDD) and the complete hypoDD locations for all 308 events are included. The color version of this figure is available only in the electronic edition.

stations considered. In contrast, the hypoDD mean and maximum coordinate error fluctuate above those for CWI confirming that the hypoDD inversion is less stable than CWI with fewer stations.

\section{Combining Arrival-Time and CWI Constraints}

In Examples 5 and 6, we compare the location of the Calaveras earthquakes using coda-wave-based and arrivaltime-based constraints independently. Because the arrivaltime (direct or difference) and coda-wave data come from different sections of the waveform they provide independent constraints on the locations. In this section we devise a location algorithm which incorporates both CWI and arrivaltime data.

We do not propose a new technique for earthquake location using arrival-time differences. Rather, we exploit the information created by hypoDD with SVD to define a probability density (or posterior) function

$$
\begin{aligned}
& P\left(\mathbf{e}_{p} \mid \Delta_{\mathrm{TT}}\right) \frac{1}{(2 \pi)^{3 / 2} \sqrt{|\Sigma|}} \\
& \quad \times \exp \left(-\frac{1}{2}\left(\left[\mathbf{e}_{p}-\mu_{\mathbf{e}_{p}}\right]^{T} \Sigma^{-1}\left[\mathbf{e}_{p}-\mu_{\mathbf{e}_{p}}\right]\right),\right.
\end{aligned}
$$

in which

$$
\mathbf{e}_{p}=\left(x_{p}, y_{p}, z_{p}\right)^{T}
$$

is the location of event $p$,

$$
\mu_{\mathbf{e}_{p}}=\left(\mu_{x_{p}}, \mu_{y_{p}}, \mu_{z_{p}}\right)^{T}
$$

is the most likely location as determined using the arrivaltime data, and 


$$
\Sigma=\left(\begin{array}{ccc}
\sigma_{x_{p}}^{2} & 0 & 0 \\
0 & \sigma_{y_{p}}^{2} & 0 \\
0 & 0 & \sigma_{z_{p}}^{2}
\end{array}\right)
$$

is the covariance matrix. We define the mean location $\mu_{\mathbf{e}_{p}}$ and covariance matrix by the hypoDD optimum solution and its uncertainties. It is important to note that hypoDD must be used with SVD to obtain useful estimates of $\sigma_{x_{p}}, \sigma_{y_{p}}$, and $\sigma_{z_{p}}$ because the errors reported by conjugate gradient methods (LSQR) are grossly underestimated in hypoDD (Waldhauser, 2001).

We pose the location problem using the negative loglikelihood

$$
\begin{aligned}
L\left(\mathbf{e}_{1}, \mathbf{e}_{2}, \ldots, \mathbf{e}_{1}, \mathbf{e}_{n}\right)= & -\sum_{i=1}^{n} \ln \left[P\left(\mathbf{e}_{i} \mid \Delta_{\mathrm{TT}}\right)\right] \\
& -\sum_{i=1}^{n-1} \sum_{j=i+1}^{n} \ln \left[P\left(\delta_{\mathrm{CWIN}} \mid \mathbf{e}_{i}, \mathbf{e}_{j}\right)\right],
\end{aligned}
$$

in which $\left(\mathbf{e}_{1}, \mathbf{e}_{2}, \ldots, \mathbf{e}_{n}\right)$ is the joint location,

$$
\sum_{i=1}^{n} \ln \left[P\left(\mathbf{e}_{i} \mid \Delta_{\mathrm{TT}}\right)\right]
$$

incorporates the arrival-time constraints and

$$
\sum_{i=1}^{n-1} \sum_{j=i+1}^{n} \ln \left[P\left(\delta_{\mathrm{CWIN}} \mid \mathbf{e}_{i}, \mathbf{e}_{j}\right)\right]
$$

incorporates the coda waves.

We must differentiate $L$ to use the Polak-Ribiere conjugate gradient technique of Press et al. (1987). The derivative of $L\left(\mathbf{e}_{1}, \mathbf{e}_{2}, \ldots, \mathbf{e}_{n}\right)$ with respect to $x_{p}$ is given by

$$
\begin{aligned}
\frac{\partial L}{\partial x_{p}}= & -\frac{\partial \ln \left[P\left(\mathbf{e}_{p} \mid t_{\mathrm{DD}}\right)\right]}{\partial x_{p}}-\sum_{i=p+1}^{N} \frac{\partial \ln \left[P\left(\delta_{\mathrm{CWIN}} \mid \mathbf{e}_{p}, \mathbf{e}_{i}\right)\right]}{\partial x_{p}} \\
& -\sum_{j=1}^{p-1} \frac{\partial \ln \left[P\left(\delta_{\mathrm{CWIN}} \mid \mathbf{e}_{j}, \mathbf{e}_{p}\right)\right]}{\partial x_{p}},
\end{aligned}
$$

in which

$$
\sum_{i=p+1}^{N} \frac{\partial \ln \left[P\left(\delta_{\mathrm{CWIN}} \mid \mathbf{e}_{p}, \mathbf{e}_{i}\right)\right]}{\partial x_{p}}
$$

and

$$
\sum_{j=1}^{p-1} \frac{\partial \ln \left[P\left(\delta_{\mathrm{CWIN}} \mid \mathbf{e}_{j}, \mathbf{e}_{p}\right)\right]}{\partial x_{p}}
$$

are defined in the Appendix, and

$$
\begin{aligned}
\frac{\partial \ln \left[P\left(\mathbf{e}_{p} \mid t_{\mathrm{DD}}\right)\right]}{\partial x_{p}}= & -\frac{1}{2}[1,0,0]^{T} \Sigma^{-1}\left[\mathbf{e}_{p}-\mu_{\mathbf{e}_{p}}\right] \\
& -\frac{1}{2}\left[\mathbf{e}_{p}-\mu_{\mathbf{e}_{p}}\right]^{T} \Sigma^{-1}[1,0,0] .
\end{aligned}
$$

Similarly, for the derivatives with respect to $y_{p}$ and $z_{p}$, we have

$$
\begin{aligned}
\frac{\partial \ln \left[P\left(\mathbf{e}_{p} \mid t_{\mathrm{DD}}\right)\right]}{\partial y_{p}}= & -\frac{1}{2}[0,1,0]^{T} \Sigma^{-1}\left[\mathbf{e}_{p}-\mu_{\mathbf{e}_{p}}\right] \\
& -\frac{1}{2}\left[\mathbf{e}_{p}-\mu_{\mathbf{e}_{p}}\right]^{T} \Sigma^{-1}[0,1,0]
\end{aligned}
$$

and

$$
\begin{aligned}
\frac{\partial \ln \left[P\left(\mathbf{e}_{p} \mid t_{\mathrm{DD}}\right)\right]}{\partial z_{p}}= & -\frac{1}{2}[0,0,1]^{T} \Sigma^{-1}\left[\mathbf{e}_{p}-\mu_{\mathbf{e}_{p}}\right] \\
& -\frac{1}{2}\left[\mathbf{e}_{p}-\mu_{\mathbf{e}_{p}}\right]^{T} \Sigma^{-1}[0,0,1] .
\end{aligned}
$$

Combining the arrival-time and coda-wave data offers two advantages. First, it combines independent constraints on the event locations offering further confidence in the resulting solution. Second, the arrival-time constraints in the form of equation (27) resolve the inherent nonuniqueness of the CWI inversion that is associated with translation, rotation, and reflection around a global coordinate system. This means that it is no longer necessary to use a local coordinate system, and we can solve directly for location with respect to a global reference. Collectively, these advantages improve the behavior of the Polak-Ribiere optimization leading to faster and more stable convergence. Consequently, we no longer need to consider multiple randomly chosen starting locations.

\section{Example 7: Combining Arrival-Time and CWI}

\section{Constraints}

Figure 11 illustrates the earthquake locations obtained when we combine the arrival-time and coda-wave data using all data (left) and five stations (right). The linear features observed in the original hypoDD inversions (see Fig. 6) are evident in both cases. However, the coda waves introduce a scatter around these streaks. That is, the locations in Figure 11 result from a trade-off between hypoDD's desire to place the events on linear features and the coda-waves voracity to push them away from streaks. When all stations are used the hypoDD constraints are strong and little offstreak scatter is introduced. As we reduce hypoDD's leverage by decreasing the number of stations to five, we observe an increase in off-streak scatter resulting from the enhanced influence of the coda.

\section{Example 8: Combining CWI and Arrival Times When the Arrival Times Constrain a Limited Number of Events}

In intraplate regions such as Australia it is common to deploy temporary seismometers to monitor aftershocks for significant events (Bowman et al., 1990; Leonard, 2002). Traditionally, these deployments facilitate a higher accuracy of location for events occurring during the deployment period. Using our combined inversion it is possible to relocate all events by employing the detailed arrival-time data when the temporary network is in situ and using coda waves 

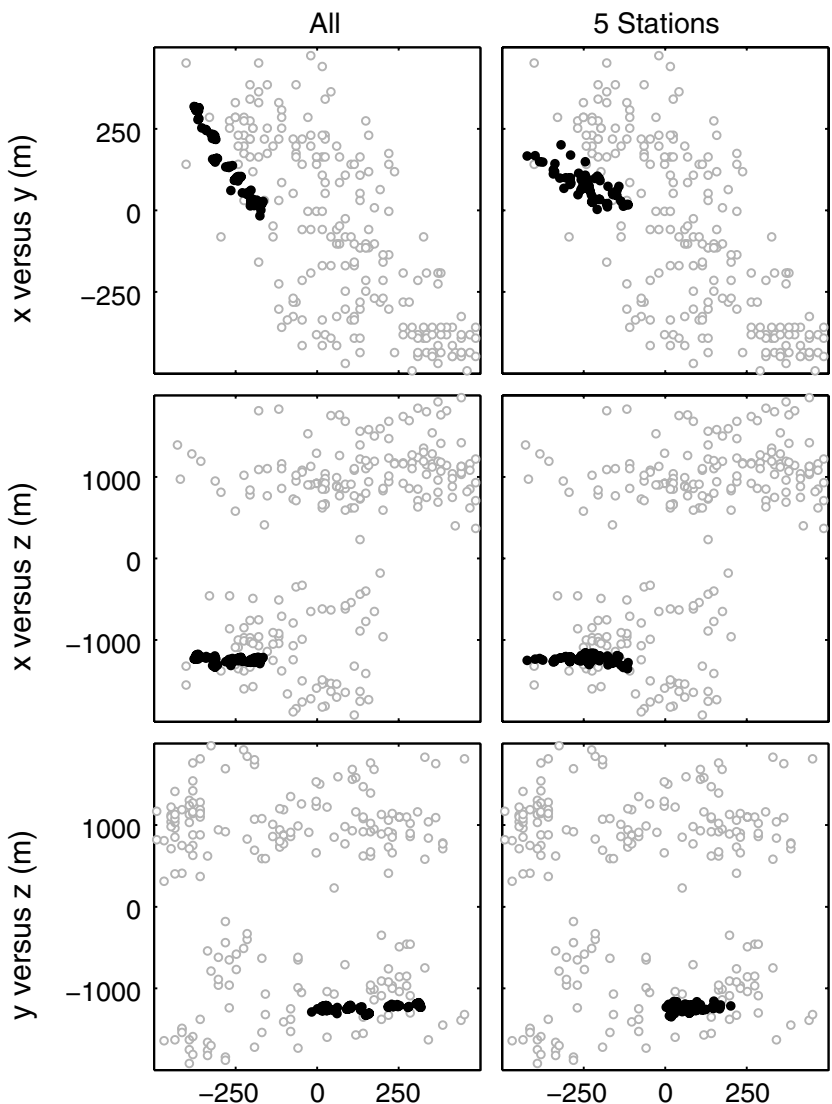

Figure 11. Example 7: Combined hypoDD (SVD) and CWI relative locations using data form all stations (left) and five stations (right). Axes as defined in Figure 6.

from network stations when the deployment is absent. The hypothesis, to be tested in this section, is that conducting such a combined inversion will improve the location accuracy of events outside the deployment period.

An estimate of the cumulative number of aftershocks $N(t)$ after $t$ days can be modeled by the modified Omori formula,

$$
N(t)=K \frac{c^{1-p}+(t+c)^{1-p}}{p-1}
$$

(Utsu et al., 1995). The empirically derived constants, $K, C$, and $p$ vary between tectonic settings. For example, using recorded aftershocks with $M \geq 3.2$ of the Hokkaido-NanseiOki, Japan, $M_{\mathrm{s}} 7.8$ earthquake of 12 July 1993, Utsu et al. (1995) obtained maximum likelihood estimates for $K, p$, and $c$ of $906.5,1.256$, and 1.433 , respectively. With these empirically derived values an array deployed within 4 days and left for 150 days will record roughly one half of the aftershocks occurring within the first 1000 days. That is,

$$
\frac{N(150+4)-N(4)}{N(1000)}=\frac{2257-934}{2626} \approx 0.5 .
$$

This is illustrated in Figure 12 which shows the best fitting Omori formula separated into segments before, during, and after the pseudotemporary deployment.

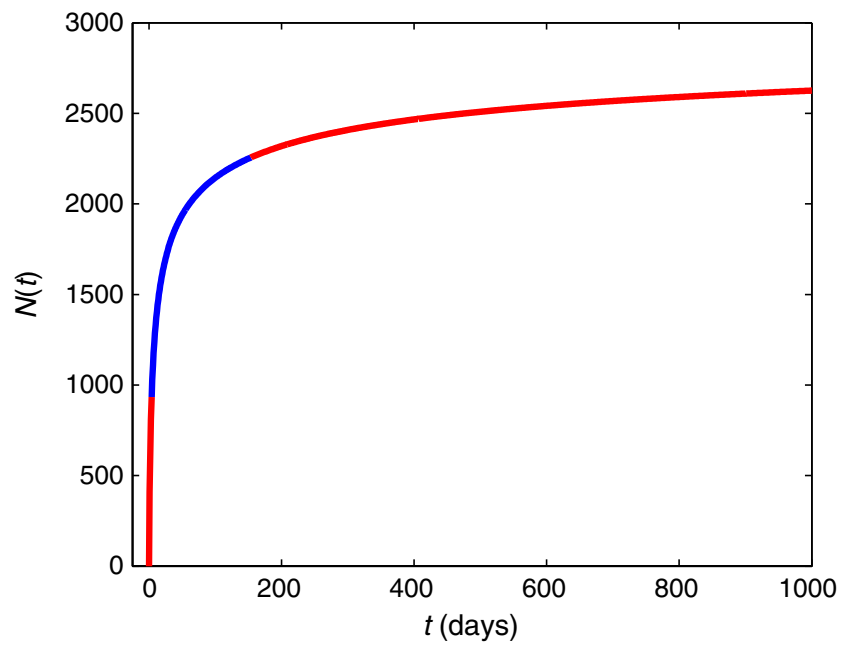

Figure 12. Modeled cumulative number of aftershocks for the Hokkaido-Nansei-Oki, Japan, $M_{\mathrm{s}} 7.8$ earthquake of 12 July 1993 using equation (35). The leftmost, middle, and rightmost segments of the line signify aftershocks occurring before, during, and after the deployment of a pseudotemporary array installed four days after the mainshock and left for 150 days. A temporary deployment of this kind will record roughly $50 \%$ of the aftershocks in the 1000 days following the mainshock. The color version of this figure is available only in the electronic edition.

With this idea of a temporary deployment in mind we have another attempt at relocating the Calaveras earthquakes. In example 8 we consider the arrival-time constraints on half (34) of the earthquakes and incorporate coda-wave data from a single station for all 68 earthquakes. The combined inversion is shown in column 1 of Figure 13. The inversion result is similar to the combined inversion when all arrival-time data are incorporated (see Fig. 11). The slight increase in scatter observed here can be explained by the events with no arrival-time constraints and the tendency of the coda to push events away from streaks.

Remarkably, the combined coda-wave and arrival-time inversion locates all 68 earthquakes to an accuracy similar to the inversions with all data. In contrast when arrival-time data are used alone it is only possible to locate the 34 events recorded by the pseudotemporary deployment. This ability of coda waves to constrain the location of events recorded by a single station creates new opportunities for understanding earthquakes in regions with limited station coverage.

\section{Discussion and Conclusions}

CWI is an emerging technique for constraining earthquake location. The technique relies on the interference between coda waves of closely located events and is hence useful for studying earthquake clusters and/or aftershock sequences. Coda-wave constraints are independent of arrival times and can be used in isolation or combination with early onset body waves. The strength of coda is that it is possible to constrain earthquake location from a single station, an outcome demonstrated most clearly by Figures 8 and 13 . 

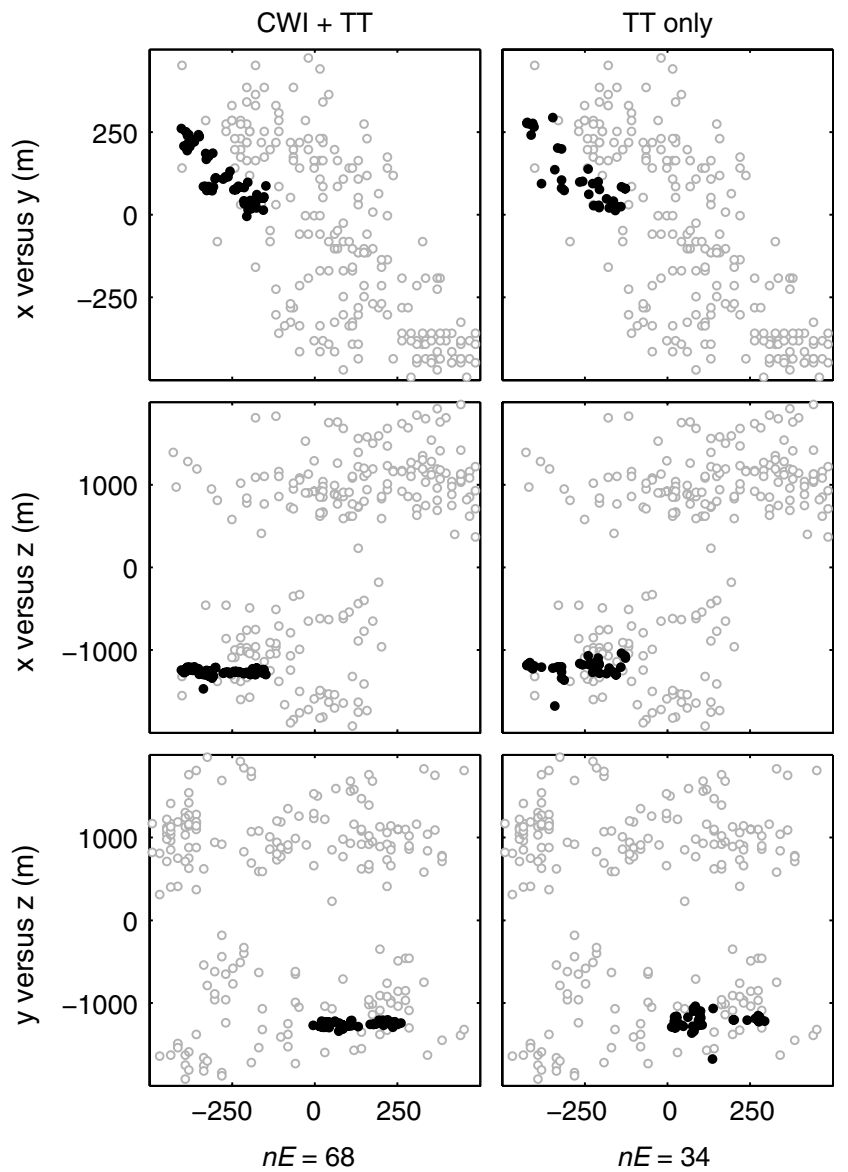

Figure 13. Example 8: Mimicking the deployment of a temporary network by ignoring data from all but station CCO for $50 \%$ (or 34 ) of the 68 events. Relative locations are shown for the combined CWI and arrival-time inversion (left) and the inversion with arrival times only (right). Only by combining the data is it possible to locate all 68 events. Furthermore, combining the data leads to a solution more consistent with Figure 6. Axes as defined in Figure 6.

CWI offers a new technique for understanding earthquakes in intraplate areas with sparse networks and poor azimuthal coverage. In particular, the ability to combine coda-wave constraints with arrival times makes it possible to link well constrained events from a temporary deployment with those recorded outside the deployment period. All that is required to achieve this is at least one network station which has recorded sufficient events from both periods. CWI facilitates the location of poorly recorded events to an accuracy approaching those recorded during the temporary deployment and therefore opens new avenues for imaging intraplate fault structures and improving our understanding of intraplate seismicity and earthquake hazard. Importantly, this analysis can be conducted for any historical aftershock sequence or earthquake swarm recorded by a temporary deployment. Our technique is, in that sense, related to the retrospective seismological observation technique of Curtis et al. (2012) that utilizes interferometry to obtain seismic signals on newly installed sensors regardless of whether the event occurs before, during, or after the physical installation of the sensor.
Another potential application of CWI is in the area of hydraulic fracturing such as hot rock geothermal projects, petroleum reservoir engineering, tight gas extraction, $\mathrm{CO}_{2}$ geosequestration, and/or underground brine injection. Monitoring pumping-induced microearthquakes is a key step in understanding the migration of fluids in such reservoirs. There is a trade-off in the ability of surface deployed networks to locate events which are small and/or deep. Downhole seismic monitoring is likely to play increasingly important roles in deep reservoir projects. CWI creates new possibilities to monitor pumping induced microearthquakes from fewer boreholes and hence dramatically reduce the costs of reservoir monitoring at large depths. It may also be possible to utilize coda for understanding hazard in tunneled mining operations where the location of deep tunnels prohibits azimuthal coverage of induced events.

\section{Data and Resources}

We thank the Northern California Earthquake Data Center (NCEDC) for providing the Calaveras data and the Northern California Seismic Network (NCSN), U.S. Geological Survey (Menlo Park), and Berkeley Seismological Laboratory (University of California, Berkeley) for contributing to the NCEDC. The waveforms can be downloaded from http://www.ncedc.org/ncedc/access.html (last accessed August 2012). We also acknowledge Felix Waldhauser and William Ellsworth, the authors of the openly available double-difference location algorithm, hypoDD, which can be downloaded from http://www.ldeo.columbia.edu/ felixw/ hypoDD.html (last accessed August 2012). The International Sesimological Centre can be found at http://www.isc.ac.uk/ (last accessed December 2012). The National Earthquake Information Center catalog can be accessed from http:// earthquake.usgs.gov/earthquakes/eqarchives/epic/ (last accessed December 2012).

\section{Acknowledgments}

Geoscience Australia, the Research School of Earth Sciences at The Australian National University, and the Center for Wave Phenomena at the Colorado School of Mines are acknowledged for supporting this research. The paper is published with permission of the CEO Geoscience Australia. Work was conducted as part of an Australian Research Council Discovery Project (DP0665111). This paper has benefited significantly from: reviews by Trevor Allen and Clive Collins at Geoscience Australia, the BSSA editorial team, Andrew Curtis from the School of GeoSciences at the University of Edinburgh, and an anonymous reviewer. The authors have also benefited from comments and advice received from Felix Waldhauser on this and earlier research on the application of CWI to earthquake location.

\section{References}

Ake, J., D. O'Connell, and L. Block (2005). Deep-injection and closely monitored induced seismicity at Paradox Valley, Colorado, Bull. Seismol. Soc. Am. 95, no. 2, 664-683.

Aki, K. (1969). Analysis of the seismic coda of local earthquakes as scattered waves, J. Geophys. Res. 74, no. 2, 615-631. 
Aster, R. C., B. Borchers, and C. H. Thurber (2005). Parameter Estimation and Inverse Problems, International Geophysics Series, Vol. 90, Elsevier Academic Press, U.S.A.

Bondár, I., S. C. Myers, E. R. Engdahl, and E. A. Bergman (2004). Epicentre accuracy based on seismic network criteria, Geophys. J. Int. 156, 483-496.

Bowman, J. R., G. Gibson, and T. Jones (1990). Aftershocks of the 1988 January 22 Tennant Creek, Australia intraplate earthquakes: Evidence for a complex thrust-fault geometry, Geophys. J. Int. 100, 87-97.

Campbell, K. W. (2003). Strong motion attenuation, in International Handbook of Earthquake and Engineering Seismology, W. H. K. Lee, H. Kanamori, P. C. Jennings, and C. Kisslinger (Editors), Vol. B, Chapter 60, Academic Press, London, 1003-1012.

Curtis, A., and R. Snieder (2002). Probing the Earth's interior with seismic tomography, in International Handbook of Earthquake Engineering Seismology, W. H. Lee, H. Kanamori, P. C. Jennings, and C. Kisslinger (Editors), Vol. A, Chapter 52, Academic Press, London, 861-874.

Curtis, A., Y. Behr, E. Entwistle, E. Galetti, J. Townend, and S. Bannister (2012). The benefit of hindsight in observational science: Retrospective seismological observations, Earth Planet. Sci. Lett. 345-348, 212-220.

Deichmann, N., and M. Garcia-Fernandez (1992). Rupture geometry from high-precision relative hypocentre locations of microearthquake clusters, Geophys. J. Int. 110, 501-517.

Douglas, A. (1967). Joint epicentre determination, Nature 215, 47-48.

Frankel, A. D., C. S. Mueller, T. P. Barnhard, E. V. Leyendecker, R. L. Wesson, S. C. Harmsen, F. W. Klein, D. M. Perkins, N. C. Dickman, S. L. Hanson, and M. G. Hopper (2000). USGS National seismic hazard maps, Earthq. Spectra 16, no. 1, 1-19.

Frèmont, M.-J., and S. D. Malone (1987). High precision relative locations of earthquakes at Mount St. Helens, J. Geophys. Res. 92, no. B10, $10,223-10,236$

Got, J.-L., J. Frèchet, and F. W. Klein (1994). Deep fault plane geometry inferred from multiplet relative relocation beneath the south flank of Kilauea, J. Geophys. Res. 99, no. B8, 15,375-15,386.

Gutenberg, B. (1945). Amplitudes of surface waves and magnitudes of shallow earthquakes, Bull. Seismol. Soc. Am. 35, 3-12.

Ito, A. (1985). High resolution relative hypocenters of similar earthquakes by cross-spectral analysis method, J. Phys. Earth 33, 279-294.

Kennett, B. L. N., E. R. Engdahl, and R. Buland (1995). Constraints on seismic velocities in the Earth from traveltimes, Geophys. J. Int. 122, 108-124.

Kennett, B. L. N., S. Fishwick, and M. Heintz (2004). Lithospheric structure in the Australian region-A synthesis of surface wave and body wave studies, Explor. Geophys. 35, 242-250.

Lees, J. M. (1998). Multiplet analysis at Coso Geothermal, Bull. Seismol. Soc. Am. 88, no. 5, 1127-1143.

Leonard, M. (2002). The Burakin WA earthquake sequence Sept 2000-June 2002, in Total Risk Management in the Privatised Era, Australian Earthquake Engineering Society Conference, M. Griffith, D. Love, P. McBean, A. McDougall, and B. Butler (Editors), Vol. 10, AEES, University of Adelaide, 22(1)-22(5).

Nadeau, R. M., and T. V. McEvilly (1997). Seismological studies at Parkfield V: Characteristic microearthquake sequences as fault-zone drilling targets, Bull. Seismol. Soc. Am. 87, no. 6, 1463-1472.

Pavlis, G. L. (1992). Appraising relative earthquake location errors, Bull. Seismol. Soc. Am. 82, no. 2, 836-859.

Press, W. H., B. P. Flannery, S. A. Teukolsky, and W. T. Vetterling (1987) Numerical Recipes: The Art of Scientific Computing, Cambridge University Press, U.S.A.

Richter, C. F. (1935). An instrumental earthquake magnitude scale, Bull. Seismol. Soc. Am. 25, no. 1, 1-32.

Robinson, D., T. Dhu, and J. Schneider (2006). Practical probabilistic seismic risk analysis: A demonstration of capability, Seismol. Res. Lett. 77, no. 4, 452-458.

Robinson, D. J., M. Sambridge, and R. Snieder (2007). Constraints on coda wave interferometry estimates of source separation: The $2.5 \mathrm{~d}$ acoustic case, Explor. Geophys. 38, no. 3, 189-199.
Robinson, D. J., M. Sambridge, and R. Snieder (2011). A probabilistic approach for estimating the separation between a pair of earthquakes directly from their coda waves, J. Geophys. Res. 116, no. B04309, 1-17, doi: 10.1029/2010JB007745.

Robinson, D. J., R. Snieder, and M. Sambridge (2007). Using coda wave interferometry for estimating the variation in source mechanism between double couple events, J. Geophys. Res. 112, no. b12302, doi: 10.1029/2007JB004925.

Rubin, A. M. (2002). Aftershocks of microearthquakes as probes of the mechanics of rupture, J. Geophys. Res. 107, no. B72142, doi: 10.1029/2001JB000496.

Rubin, A. M., D. Gillard, and J.-L. Got (1999). Streaks of microearthquakes along creeping faults, Nature 400, 635-641.

Schaff, D. P., G. H. R. Bokelmann, and G. C. Beroza (2002). Highresolution image of Calaveras Fault seismicity, J. Geophys. Res. 107, no. B92186, doi: 10.1029/2001JB000633.

Shearer, P. M. (1999). Introduction to Seismology, Cambridge University Press, U.S.A., 260 pp.

Shearer, P., E. Hauksson, and G. Lin (2005). Southern California hypocenter relocation with waveform cross-correlation, Part 2: Results using source-specific station terms and cluster analysis, Bull. Seismol. Soc. Am. 95, no. 3, 904-915, doi: 10.1785/0120040168.

Sipkin, S. A. (2002). USGS earthquake moment tensor catalog, in International Handbook of Earthquake Engineering Seismology, W. H. Lee, H. Kanamori, P. C. Jennings, and C. Kisslinger (Editors), Vol. A, Chapter 50, Academic Press, London, 823-825.

Slunga, R., S. Th. Rögnvaldsson, and R. Bödvarsson (1995). Absolute and relative locations of similar events with application to microearthquakes in southern Iceland, Geophys. J. Int. 123, no. 2, 409-419.

Snieder, R. (1999). Imaging and averaging in complex media, in Diffuse Waves in Complex Media, NATO Science Series C, J. P. Fouque, Vol. 531, Kluwer Academic Publishers, 405-454.

Snieder, R. (2006). The theory of coda wave interferometry, Pure Appl. Geophys. 163, 455-473.

Snieder, R., and M. Vrijlandt (2005). Constraining the source separation with coda wave interferometry: Theory and application to earthquake doublets in the Hayward fault, California, J. Geophys. Res. 110, no. B04301, doi: 10.1029/2004JB003317.

Spencer, C., and D. Gubbins (1980). Travel-time inversion for simultaneous earthquake location and velocity structure determination in laterally varying media, Geophys. J. Roy. Astron. Soc. 63, 95-116.

Stirling, M. W., G. H. McVerry, and K. R. Berrryman (2002). A new seismic hazard model for New Zealand, Bull. Seismol. Soc. Am. 92, no. 5, 1878-1903.

Toro, G. R., N. A. Abrahamson, and J. F. Schneider (1997). Model of strong ground motions from earthquakes in Central and Eastern North America: Best estimates and uncertainties, Seismol. Res. Lett. 68, no. 1, 41-57.

Utsu, T., Y. Ogata, and R. S. Matsu'ura (1995). The Centenary of the Omori formula for a decay law of aftershock activity, J. Phys. Earth 43, 1-33.

VanDecar, J. C., and R. Snieder (1994). Obtaining smooth solutions to large linear inverse problems, Geophysics 59, 818-829.

Waldhauser, F. (2001). hypoDD-A program to compute double-difference hypocenter locations (hypoDD version 1.0-03/2001), U.S. Geol. Surv. Open-File Rept. 01-113, Menlo Park, California.

Waldhauser, F., and W. L. Ellsworth (2000). A double-difference earthquake location algorithm: Method and application to the northern Hayward Fault, California, Bull. Seismol. Soc. Am. 90, no. 6, 1353-1368.

Waldhauser, F., and W. L. Ellsworth (2002). Fault structure and mechanics of the Hayward fault, California, from double-difference earthquake locations, J. Geophys. Res. 107, no. B3, doi: 10.1029/2000JB000084.

Waldhauser, F., and D. P. Schaff (2008). Large-scale relocation of two decades of Northern California seismicity using cross-correlation and double-difference methods, J. Geophys. Res. 133, B08311, doi: 10.1029/2007JB005479.

Waldhauser, F., W. L. Ellsworth, and A. Cole (1999). Slip-parallel lineations on the Northern Hayward Fault, California, Geophys. Res. Lett. 26, no. $23,3525-3528$. 
Appendix

\section{The Likelihood}

The likelihood $P\left(\tilde{\delta}_{\mathrm{CWIN}} \mid \tilde{\delta}_{\mathrm{t}}\right)$ used in equation (7) is given by

$$
\begin{aligned}
P\left(\tilde{\delta}_{\mathrm{CWIN}} \mid \tilde{\delta}_{\mathrm{t}}\right)= & A\left(\tilde{\delta}_{\mathrm{t}}\right) C\left(\bar{\mu}_{N}, \bar{\sigma}_{N}\right) \\
& \times \int_{0}^{\infty} B\left(\tilde{\delta}_{\mathrm{t}}, \tilde{\delta}_{\mathrm{CWI}}\right) D\left(\tilde{\delta}_{\mathrm{CWI}}, \bar{\sigma}_{N}, \bar{\mu}_{N}\right) d \tilde{\delta}_{\mathrm{CWI}},
\end{aligned}
$$

in which $\tilde{\delta}_{\mathrm{CWI}}$ is an estimate of CWI separation in the absence of noise,

$$
\begin{gathered}
A\left(\tilde{\delta}_{\mathrm{t}}\right)=\frac{1}{\left(1-\Phi_{\mu_{1}, \sigma_{1}}(0)\right) \sigma_{1} \sqrt{2 \pi}}, \\
B\left(\tilde{\delta}_{\mathrm{t}}, \tilde{\delta}_{\mathrm{CWI}}\right)=e^{\frac{-\left(\tilde{\delta}_{\mathrm{CWI}}-\mu_{1}\right)^{2}}{2 \sigma_{1}^{2}}}, \\
C\left(\bar{\mu}_{N}, \bar{\sigma}_{N}\right)=\frac{1}{\left(1-\Phi_{\bar{\mu}_{\mathrm{N}}, \bar{\sigma}_{\mathrm{N}}}(0)\right) \sigma_{\mathrm{N}} \sqrt{2 \pi}}, \\
D\left(\tilde{\delta}_{\mathrm{CWI}}, \bar{\sigma}_{N}, \bar{\mu}_{N}\right)=e^{\frac{-\left(\tilde{\delta}_{\mathrm{CWI}}-\overline{-}_{\mathrm{N}}\right)^{2}}{2 \tilde{\sigma}_{\mathrm{N}}^{2}}},
\end{gathered}
$$

and $\Phi_{\mu, \sigma}(x)$ is the cumulative Gaussian distribution function

$$
\Phi_{\mu, \sigma}(x)=\frac{1}{\sigma \sqrt{2 \pi}} \int_{-\infty}^{x} e^{\frac{-(s-\mu)^{2}}{2 \sigma^{2}}} d s
$$

(Robinson et al., 2011). The parameters $\mu_{1}$ and $\sigma_{1}$ used in equation (A2) are defined by the expressions

$$
\mu_{1}\left(\tilde{\delta}_{\mathrm{t}}\right)=a_{1} \frac{a_{2} \tilde{\delta}_{\mathrm{t}}^{a_{4}}+a_{3} \tilde{\delta}_{\mathrm{t}}^{a_{5}}}{a_{2} \tilde{\delta}_{\mathrm{t}}^{a_{4}}+a_{3} \tilde{\delta}_{\mathrm{t}}^{a_{5}}+1}
$$

and

$$
\sigma_{1}\left(\tilde{\delta}_{\mathrm{t}}\right)=c+a_{1} \frac{a_{2} \tilde{\delta}_{\mathrm{t}}^{a_{4}}+a_{3} \tilde{\delta}_{\mathrm{t}}^{a_{5}}}{a_{2} \tilde{\delta}_{\mathrm{t}}^{a_{4}}+a_{3} \tilde{\delta}_{\mathrm{t}}^{a_{5}}+1},
$$

with coefficients $a_{1}$ to $a_{5}$ and $c$ defined in Table A1. The parameters $\bar{\mu}_{\mathrm{N}}$ and $\bar{\sigma}_{\mathrm{N}}$ used in equation (A4) are obtained by finding the values that minimize the difference in a least-squares sense between the noisy CWI estimates $\tilde{\delta}_{\text {CWIN }}$ computed from the waveforms and the positively bounded Gaussian density function:

$$
P\left(\tilde{\delta}_{\mathrm{CWIN}} \mid \tilde{\delta}_{\mathrm{t}}, \tilde{\delta}_{\mathrm{CWI}}\right)=\frac{1}{\left(1-\Phi_{\bar{\mu}_{N}, \bar{\sigma}_{N}}(0)\right) \bar{\sigma}_{\mathrm{N}} \sqrt{2 \pi}} e^{\frac{-\left(\tilde{\sigma}_{\mathrm{CWIN}}-\bar{\mu}_{\mathrm{N}}\right)^{2}}{2 \overline{\mathrm{\sigma}}_{\mathrm{N}}^{2}}},
$$

with $\tilde{\delta}_{\text {CWIN }} \geq 0$.

\section{Derivatives}

The derivatives of $L\left(\mathbf{e}_{1}, \mathbf{e}_{2}, \ldots, \mathbf{e}_{\mathrm{N}}\right)$,

$$
\frac{\partial L}{\partial \hat{x}_{1}}, \frac{\partial L}{\partial \hat{y}_{1}}, \frac{\partial L}{\partial \hat{z}_{1}}, \frac{\partial L}{\partial \hat{x}_{2}}, \frac{\partial L}{\partial \hat{y}_{2}}, \frac{\partial L}{\partial \hat{z}_{2}}, \ldots, \frac{\partial L}{\partial \hat{x}_{\mathrm{N}}}, \frac{\partial L}{\partial \hat{y}_{N}}, \frac{\partial L}{\partial \hat{z}_{\mathrm{N}}},
$$

are required by the Polak-Ribiere algorithm. These are used to guide the optimization procedure towards the values of $\left(\mathbf{e}_{1}, \mathbf{e}_{2}, \ldots, \mathbf{e}_{\mathrm{N}}\right)$ which minimize $L$.

The equations for the derivatives are convoluted so we build them gradually. We start with an expression for $\delta_{t}$, the wavelength normalized separation between two events $\mathbf{e}_{p}=$ $\left(\hat{x}_{p}, \hat{y}_{p}, \hat{z}_{p}\right)$ and $\mathbf{e}_{q}=\left(\hat{x}_{q}, \hat{y}_{q}, \hat{z}_{q}\right)$ :

$$
\delta_{t}=\frac{f_{\mathrm{dom}}}{v_{s}} \sqrt{\left(\hat{x}_{p}-\hat{x}_{q}\right)^{2}+\left(\hat{y}_{p}-\hat{y}_{q}\right)^{2}+\left(\hat{z}_{p}-\hat{z}_{q}\right)^{2}}
$$

in which $f_{\text {dom }}$ is the dominant frequency of the waveforms and $V_{S}$ is the velocity between the events. Expression (A11) has derivatives

$\frac{\partial \tilde{\delta}_{\mathrm{t}}}{\partial \hat{x}_{p}}=\frac{f_{\mathrm{dom}}^{2}\left(\hat{x}_{p}-\hat{x}_{q}\right)}{v_{s}^{2} \tilde{\delta}_{\mathrm{t}}}, \quad \frac{\partial \tilde{\delta}_{\mathrm{t}}}{\partial \hat{y}_{p}}=\frac{f_{\mathrm{dom}}^{2}\left(\hat{y}_{p}-\hat{y}_{q}\right)}{v_{s}^{2} \tilde{\delta}_{\mathrm{t}}}$,

$\frac{\partial \tilde{\delta}_{\mathrm{t}}}{\partial \hat{z}_{p}}=\frac{f_{\mathrm{dom}}^{2}\left(\hat{z}_{p}-\hat{z}_{q}\right)}{v_{s}^{2} \tilde{\delta}_{\mathrm{t}}}, \quad \frac{\partial \tilde{\delta}_{\mathrm{t}}}{\partial \hat{x}_{q}}=\frac{f_{\mathrm{dom}}^{2}\left(\hat{x}_{q}-\hat{x}_{p}\right)}{v_{s}^{2} \tilde{\delta}_{\mathrm{t}}}, \quad$ and

$\frac{\partial \tilde{\delta}_{\mathrm{t}}}{\partial \hat{y}_{q}}=\frac{f_{\mathrm{dom}}^{2}\left(\hat{y}_{q}-\hat{y}_{p}\right)}{v_{s}^{2} \tilde{\delta}_{\mathrm{t}}}, \quad \frac{\partial \tilde{\delta}_{\mathrm{t}}}{\partial \hat{z}_{q}}=\frac{f_{\mathrm{dom}}^{2}\left(\hat{z}_{q}-\hat{z}_{p}\right)}{v_{s}^{2} \tilde{\delta}_{\mathrm{t}}}$.

For brevity we focus the following derivation in terms of $\hat{x}_{p}$. The remaining terms for $\mathbf{e}_{p}$ (i.e., $\hat{y}_{p}$ and $\hat{z}_{p}$ ) can be computed by following the same procedure. The derivatives for $\mathbf{e}_{q}$ can be attained by exploiting the symmetry

$$
\frac{\partial \tilde{\delta}_{\mathrm{t}}}{\partial \hat{x}_{q}}=-\frac{\partial \tilde{\delta}_{\mathrm{t}}}{\partial \hat{x}_{p}} .
$$

The chain rule gives

$$
\frac{\partial \mu_{1}}{\partial \hat{x}_{p}}=\frac{\partial \mu_{1}}{\partial \tilde{\delta}_{\mathrm{t}}} \frac{\partial \tilde{\delta}_{\mathrm{t}}}{\partial \hat{x}_{p}}
$$

in which differentiating equation (A7) gives

$$
\frac{\partial \mu_{1}}{\partial \tilde{\delta}_{\mathrm{t}}}=a_{1} \frac{a_{2} a_{4} \tilde{\delta}_{\mathrm{t}}^{a_{4}-1}+a_{3} a_{5} \tilde{\delta}_{\mathrm{t}}^{a_{5}-1}}{\left(a_{2} \tilde{\delta}_{\mathrm{t}}^{a_{4}}+a_{3} \tilde{\delta}_{\mathrm{t}}^{a_{5}}+1\right)^{2}} .
$$

Similarly, we have

$$
\frac{\partial \sigma_{1}}{\partial \hat{x}_{p}}=\frac{\partial \sigma_{1}}{\partial \tilde{\delta}_{\mathrm{t}}} \frac{\partial \tilde{\delta}_{\mathrm{t}}}{\partial \hat{x}_{p}},
$$

in which $\frac{\partial \sigma_{1}}{\partial \tilde{\sigma}_{t}}$ has the identical form as (A15) with different constants $a_{1}, a_{2}, \ldots, a_{5}$ (see Table A1).

The cumulative Gaussian distribution function (A6) is

$$
\Phi_{\mu_{1}, \sigma_{1}}(0)=\frac{1}{\sigma_{1} \sqrt{2 \pi}} \int_{-\infty}^{0} e^{\frac{-\left(s-\mu_{1}\right)^{2}}{2 \sigma_{1}^{2}}} d s,
$$


Table A1

Coefficients for Equations (A7) and (A8)

\begin{tabular}{cl}
\hline \multicolumn{1}{c}{$\mu_{1}\left(\tilde{\delta}_{\mathrm{t}}\right)$} & \multicolumn{1}{c}{$\sigma_{1}\left(\tilde{\delta}_{\mathrm{t}}\right)$} \\
\hline$a_{1}=0.4661$ & $a_{1}=0.1441$ \\
$a_{2}=48.9697$ & $a_{2}=101.0376$ \\
$a_{3}=2.4693$ & $a_{3}=120.3864$ \\
$a_{4}=4.2467$ & $a_{4}=2.8430$ \\
$a_{5}=1.1619$ & $a_{5}=6.0823$ \\
& $c=0.017$ \\
\hline
\end{tabular}

which has derivative

$$
\frac{\partial \Phi_{\mu_{1}, \sigma_{1}}(0)}{\partial \hat{x}_{p}}=\frac{\sigma_{1} \int_{-\infty}^{0} \frac{\partial g}{\partial \hat{x}_{p}} e^{g} d s-\frac{\partial \sigma_{1}}{\partial \hat{x}_{p}} \int_{-\infty}^{0} e^{g} d s}{\sigma_{1}^{2} \sqrt{2 \pi}},
$$

in which

$$
g=\frac{-\left(s-\mu_{1}\right)^{2}}{2 \sigma_{1}^{2}}
$$

and

$$
\frac{\partial g}{\partial \hat{x}_{p}}=\frac{4 \sigma_{1}^{2}\left(s-\mu_{1}\right) \frac{\partial \mu_{1}}{\partial \hat{x}_{p}}+4 \sigma_{1} \frac{\partial \sigma_{1}}{\partial \hat{x}_{p}}\left(s-\mu_{1}\right)^{2}}{4 \sigma_{1}^{4}} .
$$

Now, we have all the pieces to compute the derivatives of $A=A\left(\delta_{\mathrm{t}}\right)$ and $B=B\left(\delta_{t}, \delta_{\mathrm{CWI}}\right)$ as follows:

$$
\frac{\partial A}{\partial \hat{x}_{p}}=-\frac{-\frac{\partial \Phi_{\mu_{1}, \sigma_{1}}(0)}{\partial \hat{x}_{p}} \sigma_{1}+\left(1-\Phi_{\mu_{1}, \sigma_{1}}(0)\right) \frac{\partial \sigma_{1}}{\partial \hat{x}_{p}}}{\left(1-\Phi_{\mu_{1}, \sigma_{1}}(0)\right)^{2} \sigma_{1}^{2} \sqrt{2 \pi}}
$$

and

$$
\frac{\partial B}{\partial \hat{x}_{p}}=e^{h} \frac{\partial h}{\partial \hat{x}_{p}},
$$

in which

$$
h=\frac{-\left(\delta_{\mathrm{CWI}}-\mu_{1}\right)^{2}}{2 \sigma_{1}^{2}}
$$

and

$$
\frac{\partial h}{\partial \hat{x}_{p}}=\frac{4 \sigma_{1}^{2}\left(\delta_{\mathrm{CWI}}-\mu_{1}\right) \frac{\partial \mu_{1}}{\partial \hat{x}_{p}}+4\left(\delta_{\mathrm{CWI}}-\mu_{1}\right)^{2} \sigma_{1} \frac{\partial \sigma_{1}}{\partial \hat{x}_{p}}}{4 \sigma_{1}^{4}} .
$$

Finally, we can differentiate the likelihood for an individual event pair:

$$
\begin{aligned}
\frac{\partial P\left(\delta_{\mathrm{CWIN}} \mid \tilde{\delta}_{\mathrm{t}}\right)}{\partial \hat{x}_{p}}= & \frac{\partial A\left(\tilde{\delta}_{\mathrm{t}}\right)}{\partial \hat{x}_{p}} C\left(\bar{\mu}_{N}, \bar{\sigma}_{N}\right) \\
& \times \int_{0}^{\infty} B\left(\tilde{\delta}_{\mathrm{t}}, \tilde{\delta}_{\mathrm{CWI}}\right) D\left(\tilde{\delta}_{\mathrm{CWI}}, \bar{\sigma}_{N}, \bar{\mu}_{N}\right) d \tilde{\delta}_{\mathrm{CWI}} \\
& +A\left(\tilde{\delta}_{t}\right) C\left(\bar{\mu}_{N}, \bar{\sigma}_{N}\right) \times \int_{0}^{\infty} \frac{\partial B\left(\tilde{\delta}_{\mathrm{t}}, \tilde{\delta}_{\mathrm{CWI}}\right)}{\partial \hat{x}_{p}} \\
& \times D\left(\tilde{\delta}_{\mathrm{CWI}}, \bar{\sigma}_{N}, \bar{\mu}_{N}\right) d \tilde{\delta}_{\mathrm{CWI}},
\end{aligned}
$$

and for the logarithm we have

$$
\frac{\partial \ln \left[P\left(\delta_{\mathrm{CWIN}} \mid \delta_{\mathrm{t}}\right)\right]}{\partial \hat{x}_{p}}=\frac{1}{P\left(\delta_{\mathrm{CWIN}} \mid \delta_{\mathrm{t}}\right)} \frac{\partial P\left(\delta_{\mathrm{CWIN}} \mid \delta_{\mathrm{t}}\right)}{\partial \hat{x}_{p}} .
$$

Thus, it follows that the derivative of $L$ with respect to $\hat{x}_{p}$ is given by

$$
\begin{aligned}
\frac{\partial L\left(E_{1}, E_{2}, \ldots, E_{n}\right)}{\partial \hat{x}_{p}}= & -\sum_{i=p+1}^{N} \frac{\partial \ln \left[P\left(\delta_{\mathrm{CWIN}} \mid E_{p}, E_{i}\right)\right]}{\partial \hat{x}_{p}} \\
& +\sum_{j=1}^{p-1} \frac{\partial \ln \left[P\left(\delta_{\mathrm{CWIN}} \mid E_{j}, E_{p}\right)\right]}{\partial \hat{x}_{p}}
\end{aligned}
$$

for a uniform prior. The change of sign in the middle (i.e., to addition) accounts for the change in order of the events under the conditional. Its inclusion here assumes the correct use of $\partial \tilde{\delta}_{t} / \partial \hat{x}_{p}$ or $\partial \tilde{\delta}_{t} / \partial \hat{x}_{q}$ when evaluating the left and right terms of the summation. The derivatives shown in this section appear complicated but are in practice trivial to compute numerically. Confidence in their accuracy is enhanced by demonstrating that the optimization procedure converges to the correct solution for a number of synthetic problems in $2 \mathrm{D}$ and 3D.

Earth Monitoring and Hazard Group

Geoscience Australia

GPO Box 383

Canberra ACT 2601

Australia

david.robinson@ga.gov.au

david.robinson@anu.edu.au

(D.J.R.)

Research School of Earth Sciences

Australian National University

Canberra ACT 0200

Australia

(M.S.)

Center for Wave Phenomena and Department of Geophysics

Colorado School of Mines

Golden, Colorado 80401-1887

(R.S.)

CSIRO Earth Science and Resource Engineering

Dick Perry Avenue

Kensington WA 6151

Australia

(J.H.)
Manuscript received 7 January 2013; Published Online 5 November 2013 